\title{
An illustrative example of refactoring object-oriented source code with aspect-oriented mechanisms
}

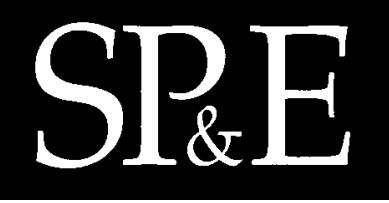

Miguel P. Monteiro ${ }^{1}$ and João M. Fernandes ${ }^{2, *, \dagger}$

${ }^{1}$ Departamento de Informática, Faculdade de Ciências e Tecnologia da Universidade Nova de Lisboa, 2829-516 Caparica, Portugal

${ }^{2}$ Departamento de Informática \& CCTC, Universidade do Minho, 4710-057 Braga, Portugal

\section{SUMMARY}

This paper describes a refactoring process that transforms a Java source code base into a functionally equivalent AspectJ source code base. The process illustrates the use of a collection of refactorings for aspect-oriented source code, covering the extraction of scattered implementation elements to aspects, the internal reorganization of the extracted aspects and the extraction of commonalities to super-aspects. Copyright $\odot 2007$ John Wiley \& Sons, Ltd.

Received 12 May 2006; Revised 29 April 2007; Accepted 1 May 2007

KEY WORDS: refactoring; aspect-oriented programming; design patterns

\section{INTRODUCTION}

Refactoring [1] and aspect-oriented programming (AOP) [2] are two techniques that contribute to deal with the problems of continuous evolution of software. This paper shows how both techniques can be combined to improve the structure of an existing system. In doing so, it also illustrates current notions of good style for AOP.

\footnotetext{
*Correspondence to: João M. Fernandes, Departamento de Informática, Universidade do Minho, 4710-057 Braga, Portugal.

†E-mail: jmf@di.uminho.pt

Contract/grant sponsor: FCT

Contract/grant sponsor: FEDER; contract/grant number: POSC/EIA/60189/2004
} 
AOP enables the modularization of crosscutting concerns (CCCs), thus diminishing the potential impact of changes on CCCs to code not directly related to such concerns. The advent of AOP brings forward the problem of how to deal with large number of existing object-oriented (OO) code bases. Experience with refactoring of $\mathrm{OO}$ software in recent years suggests that refactoring techniques can be effective in bringing concepts and mechanisms of aspect orientation to existing OO applications and frameworks. In previous work [3,4], we sought to expand the existing refactoring space for AOP, which is currently under development [5-7]. This work is based on AspectJ [8], a backwards-compatible extension to Java that supports the mechanisms of AOP. AspectJ is currently the primary representative of AOP at the level of programming languages. We undertook refactoring experiments on code bases in Java and/or AspectJ to derive interesting refactorings $[3,4,9]$.

A consequence of AspectJ's backwards compatibility with Java is that AspectJ supports multiple programming styles: those of Java and those that are based on aspect-specific mechanisms. In [4], we argue that Java's traditional OO ways to deal with CCCs should be considered bad style as more effective AspectJ-like mechanisms are available. In this light, traditional OO ways to deal with CCCs should be regarded as bad style, amenable to improvement through a process of refactoring.

In this paper, we illustrate results derived from experiments [4,9] performed on existing implementations of the Gang-of-Four (GoF) design patterns [10], coded in Java [11,12] and AspectJ [13]. To this end, the paper describes a complete refactoring process using 17 of the new refactorings documented in [9]. The process targets a Java implementation of the Observer design pattern [10], implemented in Java by Eckel [11]. The aim is to transform Eckel's implementation into the AspectJ implementation described in [13]. This process was originally characterized in the context of a validation effort for the collection of aspect-oriented (AO) refactorings documented in $[4,9]$.

The refactoring process described in this paper also illustrates how the capabilities of a programming language have a strong influence on the design of programs written in that language, and even on the very idea of what comprises a good design. The starting point of the refactoring process is an example of good Java design, created by the author of a popular Java tutorial [14]. The finishing point is generally regarded by the AspectJ community as an example of good design $[13,15]$. Nevertheless, the two designs are profoundly different. In addition, the Java implementation uses the Observable and Observer types from Java's java.util API, while the AspectJ implementation [13] relies on an internal mapping structure owned by the aspect modules. Consequently, the structural changes made during the refactoring process are very deep.

Several authors noted that AspectJ pointcuts can easily break when the code base to which they compose is modified [5,6,16], a problem that is currently known as the fragile pointcut problem [17]. As a consequence, many traditional OO refactorings can be unsound in the presence of aspects. Though the process described in this paper does use several OO refactorings from [1], the fragile pointcut problem is out of the scope of the paper and is not tackled here. We refer interested readers to $[18,19]$.

This paper is a revised and extended version of a paper presented at ICSM'05 [20]. The main additional contributions relative to the other paper are the more thorough analysis of the Observer pattern and the greater detail of the descriptions of the refactoring processes, particularly the second, alternative path (Section 6). This paper also includes a more developed discussion section and 
a survey of related work. Though this paper was written to stand on its own, an eclipse project complementing the paper is available online ${ }^{\ddagger}$.

The rest of the paper is structured as follows. Sections 2 and 3 present brief introductions to refactoring and AOP, respectively. Section 4 provides specific information on the system used as target of refactoring process. Section 5 describes the refactoring process. Section 6 describes a second refactoring process that is based on an abstract aspect from [13], comprising an alternative to the path described in the previous section. Section 7 provides a discussion and Section 8 surveys related work. Section 9 concludes the paper.

\section{REFACTORING}

Refactoring $[1,21,22]$ is a technique that aims to improve the internal structure of a software system, at the level of the source code, without changing its externally observable behaviour. A refactoring process comprises a sequence of small behaviour-preserving transformations of source code, also called refactorings. Each individual refactoring should be small to better ensure safety, but a process comprising a sequence of refactorings can yield a profound effect on the structure of a software system. Refactoring can be useful to evolve software in line with changes in environments and requirements.

Programmers have been performing ad hoc behaviour-preserving transformations for decades, though they did not call it refactoring. Only at the start of 1990s it did become the subject of formal study. The earliest works were by Opdyke and Johnson [23], who first coined the word refactoring and focused on OO frameworks, and by Griswold and Notkin [24], who focused on block-structured imperative programs and functional programs. Refactoring became widely known after the book by Fowler et al. [1] was published at the end of the 1990s. One important contribution of the book is to express notions of good style for OO source code, through collections of code smells, i.e. symptoms in source code that are indicative of opportunities to improve the structure through refactoring.

Fowler's book uses Java as the subject language and was published at a time when tool support for refactoring Java programs was not available. Tool developers responded positively to Fowler's challenge, and thanks to that present users of various integrated development environments can benefit from automated support for many of the refactorings described in Fowler's book. Beyond that, the concept of refactoring remains an effective way to express notions of style, as can be attested in the refactoring workbook [25] and the many exchanges in the mailing list ${ }^{\S}$ dedicated to the topic. The focus of this paper is centred on this approach to refactoring, applied to AOP source code.

Fowler et al. [1] noted that coverage by a set of unit tests is a prerequisite for the use of refactoring, in order to ensure that the behaviour is not changed. Likewise, the process described in Sections 5 and 6 of the paper is supported by an adapted version of a unit test originally found in Eckel's example (not shown in the paper: it can be found in the eclipse project available online). In addition,

\footnotetext{
$\ddagger$ The project can be downloaded from http://www.di.uminho.pt/ jmf/papers/ObserverExample.zip. It includes 33 complete code snapshots. The 'reusable' aspect from [13] is not placed in its original package, as the refactoring process required invasive changes on it. For that reason, the aspect is placed in the same package as the other elements of the system being refactored.

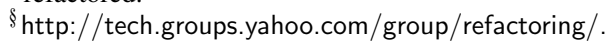


Laddad [7] proposed taking the advantage of the visualization capabilities of the AJDT plug-in for eclipse, namely the gutter annotations that show the interactions between aspects and classes. These can be checked before and after performing a refactoring. In addition, some aspect-specific techniques are proposed in [7].

\section{ASPECT-ORIENTED PROGRAMMING}

In OO systems, it often happens that certain kinds of concern, such as persistence, exception handling, logging and distribution, are scattered across the units of modularity of the system. Traditional $\mathrm{OO}$ mechanisms are unable to localize the code related to such concerns within a single module. Consequently, the representation of such concerns takes the form of multiple, small code fragments that are scattered throughout the classes of the system, a phenomenon usually referred as code scattering. Kiczales et al. [2] refer to the concerns that give rise to code scattering as CCCs. In addition, the various code fragments related to CCCs tend to be mixed with the code related to the primary functionality of the system's existing modules, harming the comprehensibility and ease of evolution of all concerns involved. This negative effect is dubbed code tangling by Kiczales et al. [2]. The implementation of a number of design patterns are examples of CCCs [13], including Observer [10], the pattern surveyed in Section 4.

$\mathrm{AOP}[2,26,27]$ is a new programming paradigm providing constructs explicitly devoted to localize source code-related CCCs in their own units of modularity-called aspects [2] - thus, eliminating code scattering and tangling. Currently, the most mature AOP language is AspectJ [8,28-30], an extension to Java that supports AOP. In the remainder of this section we briefly describe some of the novel mechanisms of AspectJ that are used in the refactoring process described in the paper.

In AspectJ, aspects are class-like modules that can hold state and behaviour and are provided with novel mechanisms through which aspects compose their functionality to multiple, scattered points of a given system. The most important mechanisms are based on the concept of joinpoint, i.e. events that occur during the execution of a program, namely method calls, constructor executions and accesses to instance fields. AspectJ provides a construct called pointcut able to capture a set of joinpoints related to non-contiguous points in the source code base. For instance, Listing 1 shows a pointcut capturing all calls to the public methods of java.io.PrintStream having any number of arguments, void as return type, and a name starting with 'print'.

In the example, the pointcut designator (PCD) call is used to capture the methods calls of interest. AspectJ provides a rich set of PCDs that includes a number of PCDs that restrict the set of captured joinpoints. In addition, PCDs can also be composed like predicates, using operators \&\&, $\|$ and !, which express set union, set intersection and set complement respectively. Listing 2 shows a pointcut similar to that of Listing 1, but complemented with a within PCD that restricts captured calls to those that originate within the lexical boundary of class Capsule.

Aspects also include advice, i.e. nameless block-statement constructs that implicitly execute upon the occurrence of each specified joinpoint. Advice can run before, after or instead of each captured

public pointcut allcalls2systemoutPrints () : call (public void java.io.PrintStream.print*(..)) ;

Listing 1. Example of an Aspect pointcut.

Copyright (C) 2007 John Wiley \& Sons, Ltd.

Softw. Pract. Exper. 2008; 38:361-396 DOI: $10.1002 /$ spe 


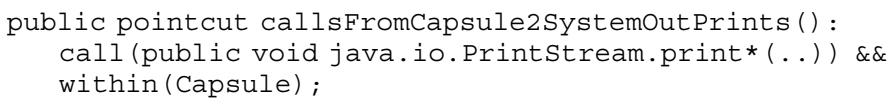

Listing 2. Example of a composition of two pointcut designators.

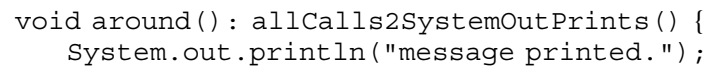

Listing 3. Example of an Aspect around advice.

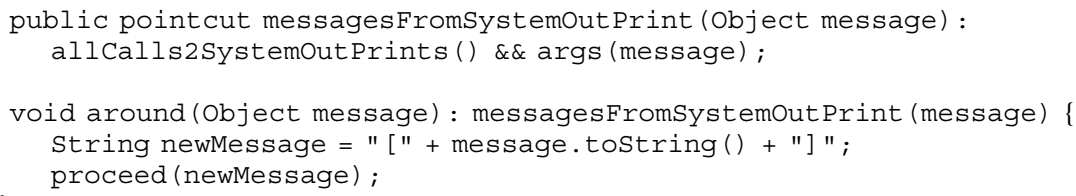

Listing 4. Example of a pointcut capturing corner from the jointpoints.

private boolean Server. disabled = false;

Listing 5. Example of an inter-type declaration.

joinpoint. The latter are called around advice. Listing 3 shows a piece of around advice that executes upon each method call captured.

The instructions within the advice from Listing 3 are executed instead of the captured joinpoints. This ability to execute blocks of statements instead of the original joinpoints (as in Listing 3) means that aspects can delete, or at least circumvent, the existing behaviour of a target code base. AspectJ also provides the keyword proceed through which around advice can execute the original, captured joinpoint. In addition, pointcuts can also capture context data from the captured joinpoints. For instance, Listing 4 shows a pointcut similar to that of Listing 1 that also captures the argument to the print method. In doing so, it also restricts the set of captured joinpoints to those calls that receive one argument of the specified type. The around advice from Listing 4 is implicitly called upon each of the joinpoints captured by the new pointcut and binds the method argument to variable message. Thus, the advice shown in Listing 4 adds square brackets to the beginning and end of the messages sent to the console.

The above composition mechanisms are often dubbed dynamic crosscutting. In addition, aspects also provide static crosscutting in the form of inter-type declarations, i.e. the ability of aspects to introduce additional state and behaviour to a set of target classes. Though the declarations are placed within the aspects at the source level, the target classes are the owners of the introduced members at the binary level. For instance, the inter-type declaration in Listing 5 declares that every instance of class Server has additional field disabled, of type boolean, initialized to false. Similar declarations can be made of methods. The visibility of inter-type members is relative to the aspect, not to target classes. When an aspect declares an inter-type member as private, only code within the aspect 
declare warning: allCalls2systemOutPrints () \&\&

!within(DeclaringAspect): "Do not use System.out.print* () .";

Listing 6. Example of a declare warning.

can use those members, despite the fact that the target classes are the owners. This further ensures the modularity of the concern related to the aspect.

In addition, AspectJ provides constructs through which the compiler is configured to generate additional error and warning messages. The constructs are clauses declare warning and declare error and accept a pointcut stating the joinpoints that give rise to the warning or error, and the associated message. For example, Listing 6 shows a declare warning that reuses the pointcut shown in Listing 1 and generates a warning upon every call to methods from System.out whose name starts with 'print' and that are not issued from the module named DeclaringAspect.

Finally, AspectJ provides the declare parents construct through which classes are made to implement additional interfaces or extend a different superclass, subject to restrictions derived from the type rules of Java. A popular idiom based on the use of interfaces comprises declaring a marker interface that represents an abstract role. In many cases the marker interface can be declared within the aspect as an inner interface, often with private visibility, ensuring that no code outside the aspect depends on the role represented by the interface. On this basis, the aspect uses inter-type declarations to add state and behaviour to the interface and uses a declare parents clause to make concrete classes implement it. Thus, the aspect composes extra functionality to a number of target classes in a transparent way. In some cases, it is feasible to separate the case-specific parts of the aspect code from the generally applicable parts, in which case an abstract base aspect can be extracted that is potentially reusable. This design technique is widely used in the examples presented in [13], including the AspectJ implementation of Observer that we describe in detail in Section 4.4.

\section{THE OBSERVER DESIGN PATTERN}

The intent of Observer (also known as Publish-Subscribe) is to 'define a one-to-many dependency between objects so that when one object changes state, all its dependents are notified and updated automatically' [10]. The pattern is an example of a CCC connecting sets of otherwise unrelated classes, implemented as a simple framework. Observer defines the role of subject for objects generating events of interest to other objects, which play the role of observer. Many implementations provide subjects with an extra field holding the list of their registered observers. Observers are registered (i.e. added to the list) by an attach operation and are removed from the list by a detach operation. When a subject gives rise to an interesting event-usually a change in its state-it calls a notify operation, which in turn calls the update operation of each registered observer. The general structure of Observer is presented in Figure 1, which is similar to that used in [10].

The example in the GoF book [10] is coded in $\mathrm{C}++$ and is therefore based on abstract classes to represent the roles of subject and observer. With Java, it is usual to represent this kind of role with interfaces. In each case, the implementing classes are called concrete subject and concrete observer, respectively.

Each observer defines its reaction to a notification in the update operation. What qualifies as an interesting event is determined by the calls to update that subjects make, so programmers must 


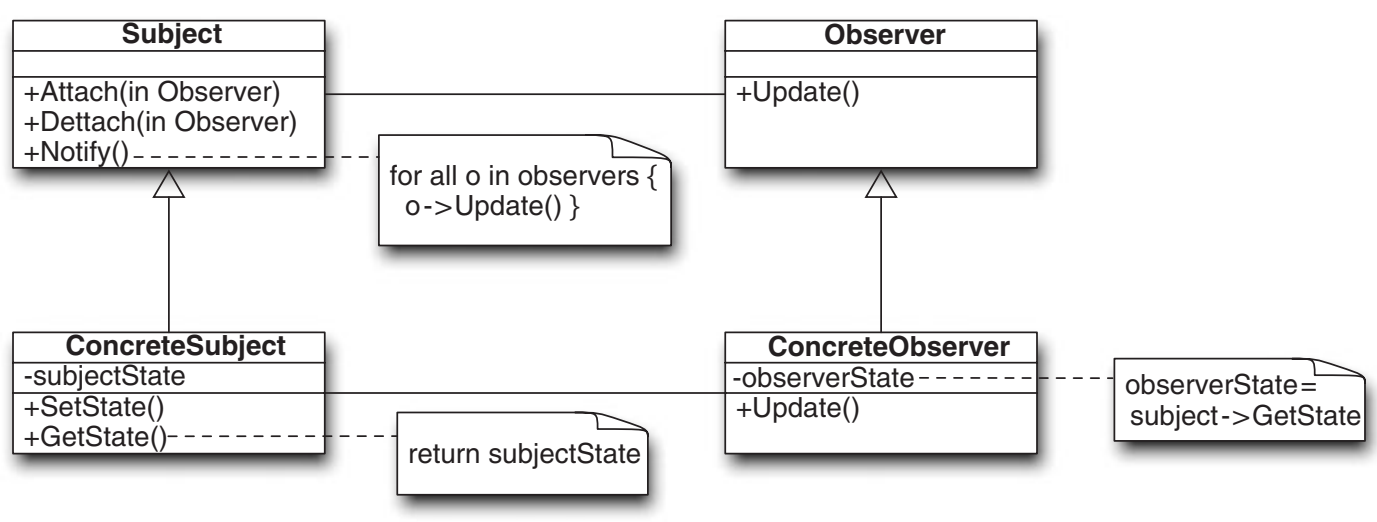

Figure 1. General structure of the observer design pattern.

ensure that such calls are placed in all desired points. In large systems, this may result in many calls, scattered throughout lots of packages. This is probably one of the reasons why implementing the pattern in large systems can be error prone [31]. Switching from one scattered implementation to another in large systems is a tedious and risky task.

\subsection{The Flower example of Observer}

The subject in Eckel's example [11] is one instance of a class representing a flower. Its interesting events are the two operations it provides: open its petals and close them. These are observed by one instance each of two unrelated types: bees and humming birds. When the flower opens its petals, its observers have breakfast. When the flower closes its petals, its observers go to sleep. These reactions are represented by simple messages sent to the console. Each of the flower operations gives rise to a different observing relationship, as observers react differently to the two events and it is possible to support one relationship without supporting the other. Eckel's example also ensures that observers only react once to each operation, i.e. if the flower executes the open operation twice with no close in between, observers are notified only upon the first open. Note that the order with which observers are notified is not relevant ${ }^{\mathbb{t}}$.

\subsection{The Java standard Observer-Observable API}

The java.util API of Java provides a ready-made implementation of Observer, comprising interface Observer and class Observable. Observer classes must implement Observer, which declares an update method. Subject classes must inherit from Observable, which provides the logic to manage the list of registered observers. Subject objects notify their observers of an interesting event by calling method notifyObservers, which is overloaded with the following

\footnotetext{
IThe javadoc documentation for method Observable.addObserver(), used by Eckel in his implementation, states that 'the order in which notifications will be delivered to multiple observers is not specified'.
} 
signatures:

public void notifyobservers (Object arg)

public void notifyobservers() // equivalent to notifyobservers (null)

This method only notifies the registered observers if the object was previously marked as having been changed, by executing the setChanged method. Observer declares method update, with the following signature:

void update (Observable o, object arg)

Subjects can use the second parameter of type Object to pass data to its observers. In order to be general purpose, the parameter must accept any type, which in Java means java.lang. Object. This provides the necessary flexibility but has the disadvantage of placing this parameter outside the reach of the type checker"l. It is the programmer's responsibility to ensure that subjects and its observers use the runtime types consistently. Method update contains the actions to be carried out by observer objects when they are notified of an interesting event. What qualifies as 'interesting' is exclusively determined by the places where calls to notify are made. Programmers using the protocol must ensure that such calls are made in all suitable places. This includes calling method setChanged. In large systems, this may comprise a large number of calls, scattered throughout lots of packages. Naturally, this has the consequence that it would be hard to switch to a different implementation after the structure is in place (for instance, one that does not require calls to a setChanged() method).

In addition to the usual problems of code scattering and tangling [2], this solution has the following disadvantages:

- Subject classes loose the option of inheriting from another class**, as they already inherit from java.util.Observable. Observer participants are less limited because they merely implement interface java.util.Observer, but this contributes to clutter their implements clause with an interface not related to the primary role of the class.

- Inheriting from java.util.Observable increases the memory footprint of each instance. Objects playing this role must carry the extra state throughout their entire life cycle, even if they only use it during certain phases.

- Use of inheritance also means that all instances will carry the extra state, even if only a subset of the instances participates in observing relationships.

- The mechanism does not provide appropriate support for multiple separate observing relationships. If instances of a class play the subject role in various observing relationships, their observers will be notified of the events relating to all of them, and need to run extra logic to distinguish one kind of event from others. It is possible to ameliorate this problem by making the subject pass itself as argument in the version of notifyObservers with two arguments, but this merely pushes the filtering logic from subjects to observers.

\footnotetext{
\|Java 5 supports generic types but the Observer/Observable protocol was not updated to support generics.

** It is fair to note that this limitation does not apply to all possible Java implementations of Observer. The implementation described in Section 4.3 manages to circumvent it.
} 


\subsection{The original pure Java implementation}

Listing 7 presents class Flower and Listing 8 presents class Bee (Hummingbird is similar) as written by Eckel [11]. Eckel's design partially circumvents the limitations mentioned in the previous section. The design relies on inner classes to isolate, within each class, the code related to the pattern. Instead of directly extending the Observer or Observable types, each participant encloses an inner class either extending Observable or implementing Observer. Each participant contains one inner class for each of the observing relationships.

Inner classes provide users of Java a limited form of multiple inheritance, in that inner classes can refer to the members of its enclosing class, even private ones. Inner classes are still free to

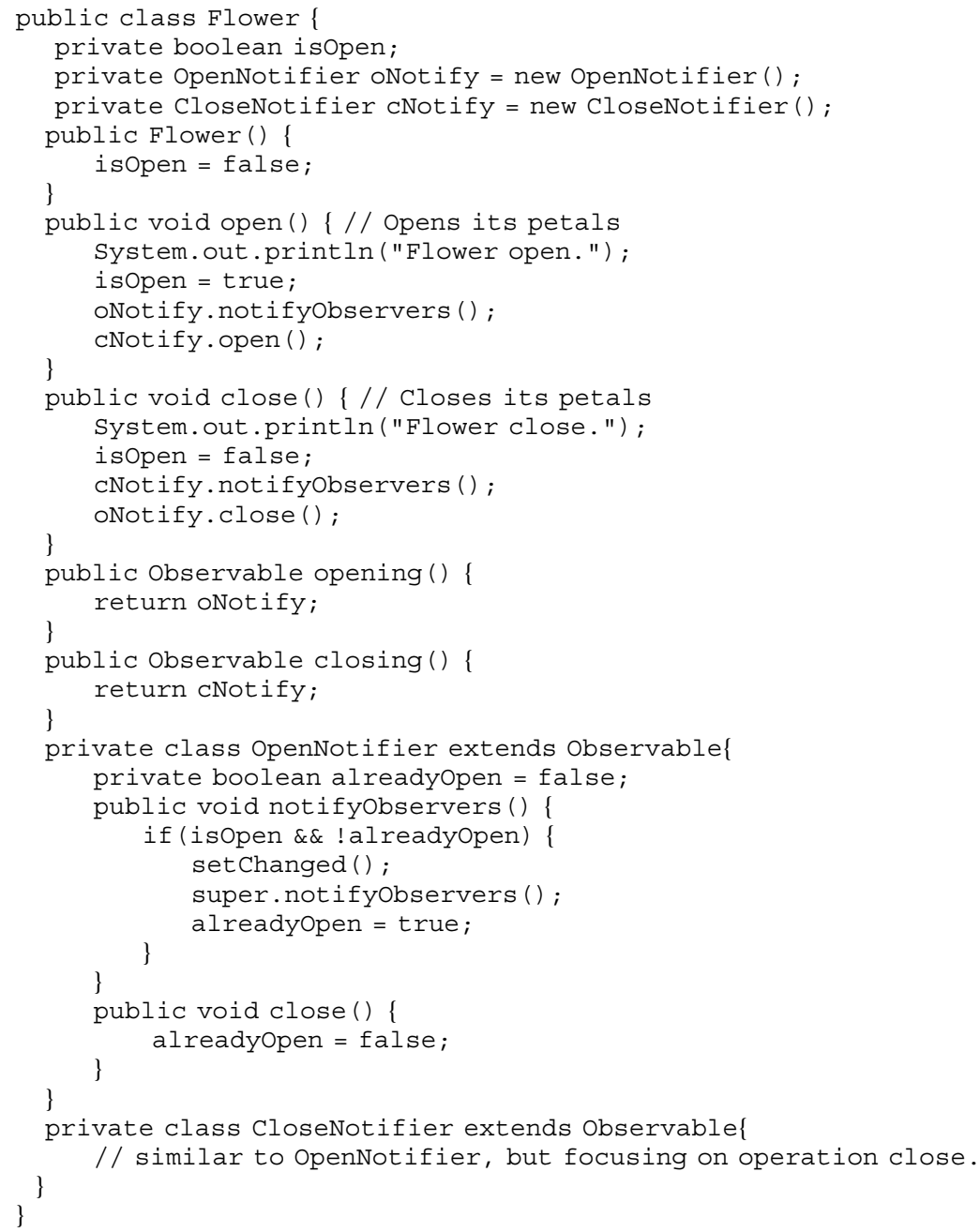

Listing 7. Initial form of the subject class Flower, as presented in [11]. 
inherit from some other class, so they enable the enclosing class to make a part of itself inherit from a given class while remaining free to use the more traditional use of single inheritance. Eckel uses this mechanism in both the subject and the observer participants, with the benefit of giving subjects the option to inherit from some useful class other than java.util.Observable (though this particular example does not take advantage of this). It also avoids cluttering the observer's implements clause with an additional interface. The design manages to localize, within each class, the code related to the pattern, but nevertheless it results in a tight structural relationship between participants and the roles they play in the pattern. It should be noted that the use of inner classes requires additional refactoring steps, as shown in Section 5.1.

What this clever design cannot achieve is obliviousness [32] from pattern roles. Participant classes betray the Double Personality smell [4], i.e. each participant plays more than one role in the system and therefore contains code related to more than one concern. This is a form of code tangling. Any method of the subject-Flower (Listing 7)—performing an interesting operation must still include code related to its role in the pattern. There is also code scattering: code dealing with the pattern is not modularized and each participant contains one inner class for each of the observing relationships. There is duplication (i.e. the Duplicated Code smell [1]), which is particularly noticeable in the two observers-Bee (Listing 8) and Hummingbird-which use four inner classes between them. Each class duplicates the code related to the two observing relationships and each observing relationship requires a duplication of essentially the same logic.

The instance of Flower plays the role of subject. Instances of Bee and Hummingbird are observers. Each observing relationship must monitor both operations, due to the requirement that observers only react to the first of multiple consecutive occurrences of the same operation (as is the case with the unit test from Eckel's example [11]). Therefore, observers of open need to be notified of close, to determine whether a call to open belongs to a sequence of calls to open without calls to close in between. This applies to observations of both open and close.

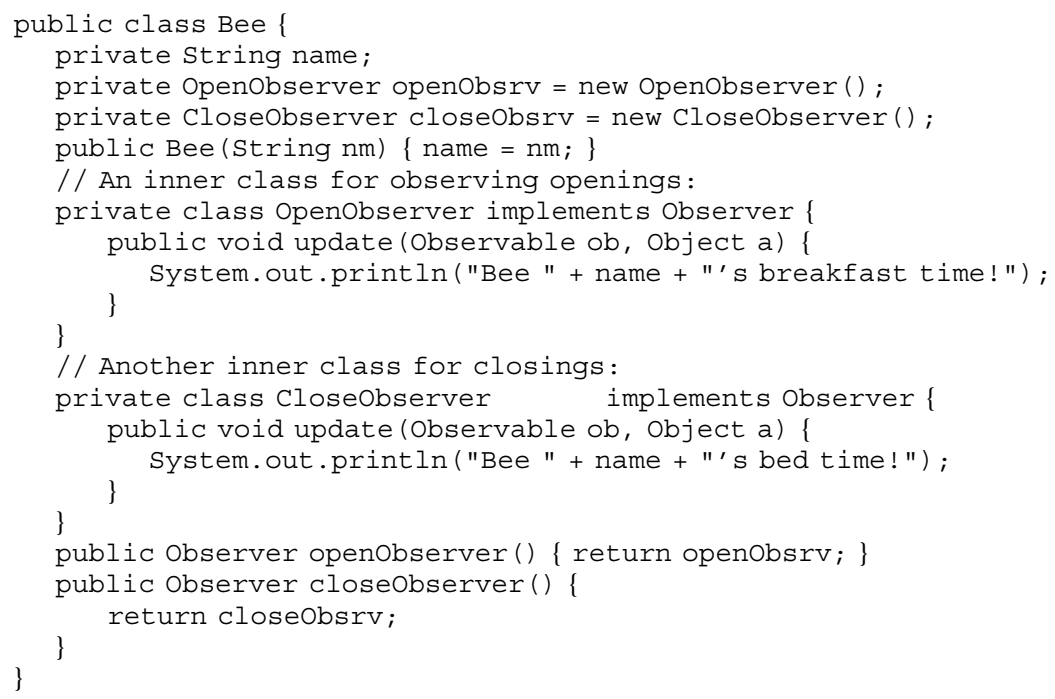

Listing 8. Initial form of observer class Bee. 


\subsection{The reusable AO implementation of Observer}

The AspectJ implementation proposed in [13] comprises an abstract base aspect-ObserverProtocol (Listing 9) - that deals with the parts common to all cases, plus concrete sub-aspects that deal with case-specific parts (Listing 10). The common parts comprise the following:

- The subject and observer roles, modelled by the inner marker interfaces Subject and Observer.

- Maintenance of a mapping from subjects to observers, implemented with a hash table field (perSubjectObservers), owned by instances of the aspect.

- The update logic, in which changes in the subject trigger updates in the observers. Changes in subject state are modelled by abstract pointcut subjectChange.

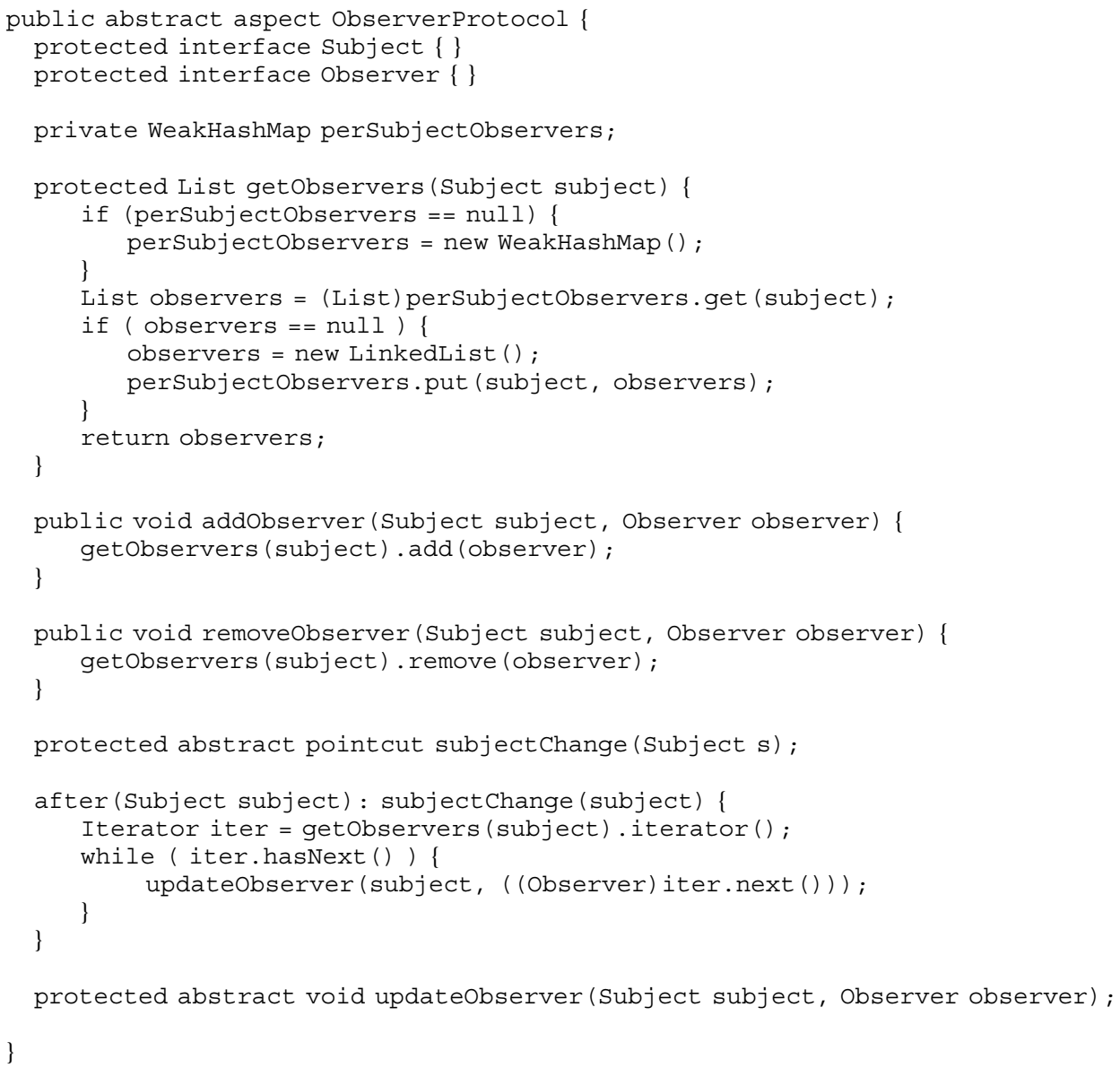

Listing 9. Reusable aspect implementation of Observer from [13]. 


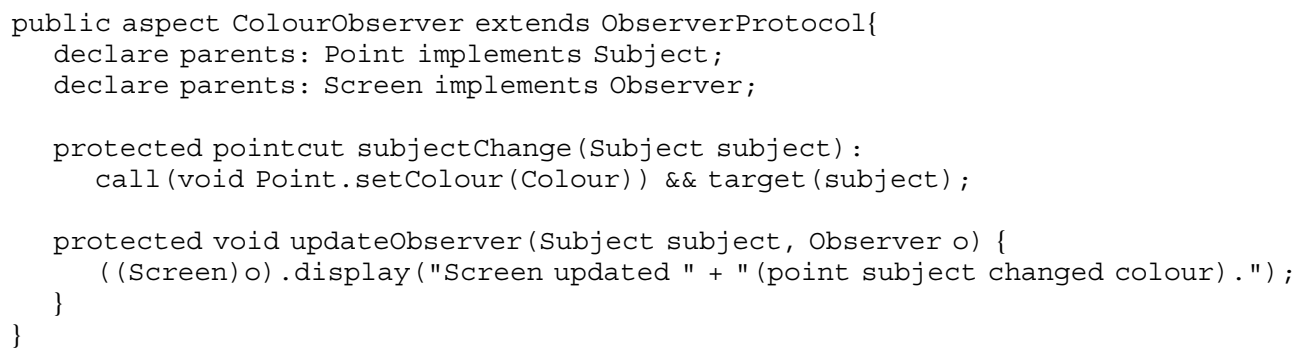

Listing 10. Example of case specific, concrete aspect reusing ObserverProtocol.

Parts specific to individual cases are:

- Assignment of roles subject and observer to concrete classes implemented through declare parents clauses.

- Changes to the subject that are of interest to its observers implemented by a definition of abstract pointcut subjectChange.

- The logic to update observers at appropriate points, implemented by aspect method updateObserver.

Participant classes in the AspectJ implementation are oblivious to the pattern roles. None of the disadvantages mentioned in relation to the Java implementation applies to this case. Participant classes remain free to inherit from other classes, and instances do not expend additional memory when not participating in observing relationships. Note that the mapping between a subject and its observers is maintained by the aspect itself, rather than being supported by means of inter-type declarations, as it could be expected from a more straightforward implementation: if the aspect resorted to inter-type declarations, all instances of the target classes would be affected, throughout their entire life cycles. The structure managing the mappings is defined in ObserverProtocol, so each concrete sub-aspect has its own instance of the hash map. One disadvantage of this implementation is a possible degradation in performance, when dealing with large systems comprising large numbers of participant objects (no performance measurement is presented in [13]).

\section{THE REFACTORING PROCESS}

Tables I-III present the 17 refactorings from [4,9] that are used throughout the refactoring process. As with most collections of refactorings, each individual refactoring should be small to better ensure safety. The process illustrated in this section is carried out according to the strategy proposed in $[4,9]$ for the extraction of a CCC. The strategy establishes that prior to anything else, all elements related to the target concern should be moved to a single module, using refactorings from Table I. After the extraction is completed, the focus should be on improving the internal structure of the extracted aspects, using the refactorings from Table II. The kind of improvements we describe are considerably easier to perform (or possible at all) after the associated implementation is modularized. 
Table I. Refactorings to extract crosscutting concerns into aspects.

\begin{tabular}{|c|c|c|}
\hline Name & Typical situation & Recommended action \\
\hline Extract Feature into Aspect & $\begin{array}{l}\text { Code related to a feature is scat- } \\
\text { tered across multiple methods and } \\
\text { classes, tangled with unrelated } \\
\text { code }\end{array}$ & $\begin{array}{l}\text { Extract to an aspect all imple- } \\
\text { mentation elements related to the } \\
\text { feature }\end{array}$ \\
\hline Extract Fragment into Advice & $\begin{array}{l}\text { Part of a method is related to } \\
\text { a concern whose code is being } \\
\text { moved to an aspect }\end{array}$ & $\begin{array}{l}\text { Create a pointcut capturing the } \\
\text { appropriate joinpoint and context } \\
\text { and move the code fragment to } \\
\text { an advice based on the pointcut }\end{array}$ \\
\hline Extract Inner Class to Standalone & $\begin{array}{l}\text { An inner class relates to a concern } \\
\text { being extracted into an aspect }\end{array}$ & $\begin{array}{l}\text { Eliminate dependencies from the } \\
\text { enclosing class and turn the inner } \\
\text { class into a stand-alone class }\end{array}$ \\
\hline Inline Interface within Aspect & $\begin{array}{l}\text { One or several interfaces are used } \\
\text { only by an aspect }\end{array}$ & $\begin{array}{l}\text { Move the interfaces to within the } \\
\text { aspect }\end{array}$ \\
\hline Move Field from Class to Inter-type & $\begin{array}{l}\text { A field relates to a concern other } \\
\text { than the primary concern of its } \\
\text { owner class }\end{array}$ & $\begin{array}{l}\text { Move the field from the class to } \\
\text { the aspect as an inter-type decla- } \\
\text { ration }\end{array}$ \\
\hline $\begin{array}{l}\text { Move Method from Class to Inter- } \\
\text { type }\end{array}$ & $\begin{array}{l}\text { A method belongs to a concern } \\
\text { other than the primary concern of } \\
\text { its owner class }\end{array}$ & $\begin{array}{l}\text { Move the method into the aspect } \\
\text { that encapsulates the secondary } \\
\text { concern as an inter-type declara- } \\
\text { tion }\end{array}$ \\
\hline $\begin{array}{l}\text { Replace Implements with Declare } \\
\text { Parents }\end{array}$ & $\begin{array}{l}\text { Classes implement an interface } \\
\text { related to a secondary concern. } \\
\text { Class code implementing the } \\
\text { interface is used only when the } \\
\text { secondary concern is included in } \\
\text { the system build }\end{array}$ & $\begin{array}{l}\text { Replace the implements in the } \\
\text { class with a equivalent declare } \\
\text { parents in the aspect }\end{array}$ \\
\hline
\end{tabular}

Some of the refactorings from Table II serve to remove the Aspect Laziness smell [4], i.e. situations when an aspect statically introduces state and behaviour to a set of classes when a more dynamic and unpluggable composition is desirable. This smell can be found in systems that require such flexibility of composition and yet resort to inter-type declarations, which performs the compositions statically. Those refactorings serve to replace this static mapping with a similar mapping programmatically supported by the aspect. Figure 2 illustrates the structural effects performed by those refactorings. The refactorings responsible for replacing the inter-type declarations with a mapping of additional state and behaviour are Replace Inter-type Field with Aspect Map and Replace Inter-type Method with Aspect Method. Their effect is illustrated in Figure 3. For more details, see [4,7].

Finally, we deal with duplication between multiple aspects by factoring out commonalities to a (in this case reusable) super-aspect, by using the refactorings from Table III. Figure 3 illustrates the structural effects of this group of refactorings. Note that Figure 3 mentions all refactorings from this group, not just the ones used in the example of this paper.

From a certain point, the described process follows two alternative paths, both ending with the AOP design described in Section 4.4. The first path is performed solely in terms of the original code, and the second path adds to the system, at a certain point, the abstract base aspect from [13] (ObserverProtocol). Sections 5.1-5.3 describe the first path in detail, comprising the three phases 
Table II. Refactorings to restructure the internals of aspects obtained through extraction processes.

\begin{tabular}{|c|c|c|}
\hline Name & Typical situation & Recommended action \\
\hline $\begin{array}{l}\text { Extend Marker } \\
\text { Interface with } \\
\text { Signature }\end{array}$ & $\begin{array}{l}\text { An inner interface models a role used within } \\
\text { the aspect. You would like the aspect to call } \\
\text { a method specific to a type that implements } \\
\text { the interface but that is not declared by it }\end{array}$ & $\begin{array}{l}\text { Add an inter-type abstract declaration of } \\
\text { the case-specific method signature to the } \\
\text { interface }\end{array}$ \\
\hline $\begin{array}{l}\text { Generalise Target } \\
\text { Type with Marker } \\
\text { Interface }\end{array}$ & $\begin{array}{l}\text { An aspect refers to case-specific concrete } \\
\text { types, preventing it from being reusable }\end{array}$ & $\begin{array}{l}\text { Replace the references to specific types } \\
\text { with a marker interface and make the } \\
\text { specific types implement the marker } \\
\text { interface }\end{array}$ \\
\hline $\begin{array}{l}\text { Introduce Aspect } \\
\text { Protection }\end{array}$ & $\begin{array}{l}\text { You would like an inter-type member to be } \\
\text { visible within the declaring aspect and all its } \\
\text { sub-aspects, but not outside the aspect } \\
\text { inheritance chain }\end{array}$ & $\begin{array}{l}\text { Declare the inter-type member as public } \\
\text { and place a declare error preventing its } \\
\text { use outside the aspect inheritance chain }\end{array}$ \\
\hline $\begin{array}{l}\text { Replace Inter-type } \\
\text { Field with Aspect } \\
\text { Map }\end{array}$ & $\begin{array}{l}\text { An aspect statically introduces additional } \\
\text { state to a set of classes, when a more } \\
\text { dynamic or flexible link between state and } \\
\text { targets would be desirable. }\end{array}$ & $\begin{array}{l}\text { Replace the inter-type declarations with a } \\
\text { structure owned by the aspect that } \\
\text { performs a map between the target objects } \\
\text { and the additional state }\end{array}$ \\
\hline $\begin{array}{l}\text { Replace Inter-type } \\
\text { Method with } \\
\text { Aspect Method } \\
\text { Tidy Up Internal } \\
\text { Aspect Structure }\end{array}$ & $\begin{array}{l}\text { An aspect introduces additional methods to } \\
\text { a class or interface, when a more dynamic } \\
\text { and flexible composition would be desirable } \\
\text { The internal structure of an aspect resulting } \\
\text { from the extraction of a crosscutting concern } \\
\text { is sub-optimal }\end{array}$ & $\begin{array}{l}\text { Replace the inter-type method with an } \\
\text { aspect method that gets the target object as } \\
\text { an extra parameter } \\
\text { Tidy up the internal structure of the aspect } \\
\text { by removing duplicated inter-type } \\
\text { declarations and dependencies on case- } \\
\text { specific target types }\end{array}$ \\
\hline
\end{tabular}

Table III. Refactorings to improve the generalisation of aspects.

\begin{tabular}{|c|c|c|}
\hline Name & Typical situation & Recommended action \\
\hline $\begin{array}{l}\text { Extract } \\
\text { Super-aspect }\end{array}$ & $\begin{array}{l}\text { Two or more aspects contain similar code } \\
\text { and functionality }\end{array}$ & $\begin{array}{l}\text { Move the common features to a super- } \\
\text { aspect }\end{array}$ \\
\hline $\begin{array}{l}\text { Pull Up Marker } \\
\text { Interface }\end{array}$ & $\begin{array}{l}\text { All sub-aspects use a marker interface to } \\
\text { model the same role }\end{array}$ & $\begin{array}{l}\text { Move the marker interfaces to the super- } \\
\text { aspect }\end{array}$ \\
\hline Pull Up Pointcut & All sub-aspects declare identical pointcuts & Move the pointcuts to the super-aspect \\
\hline Push Down Advice & $\begin{array}{l}\text { A piece of advice is used by only some sub- } \\
\text { aspects, or each sub-aspect requires different } \\
\text { advice code }\end{array}$ & $\begin{array}{l}\text { Move the advice to the sub-aspects that } \\
\text { use it }\end{array}$ \\
\hline
\end{tabular}

of the refactoring strategy, each relating to a composite refactoring [4] prescribing the use of other refactorings:

1. Extract Feature into Aspect: extracts the two observing relationships into aspects.

2. Tidy Up Internal Aspect Structure: improves the internal structure of the extracted aspects.

3. Extract Super-aspect: factors out common code from the aspects to an abstract super-aspect.

The above three refactorings are composite refactorings: they are more general than most and their purpose is to set the background for the use of other refactorings. 


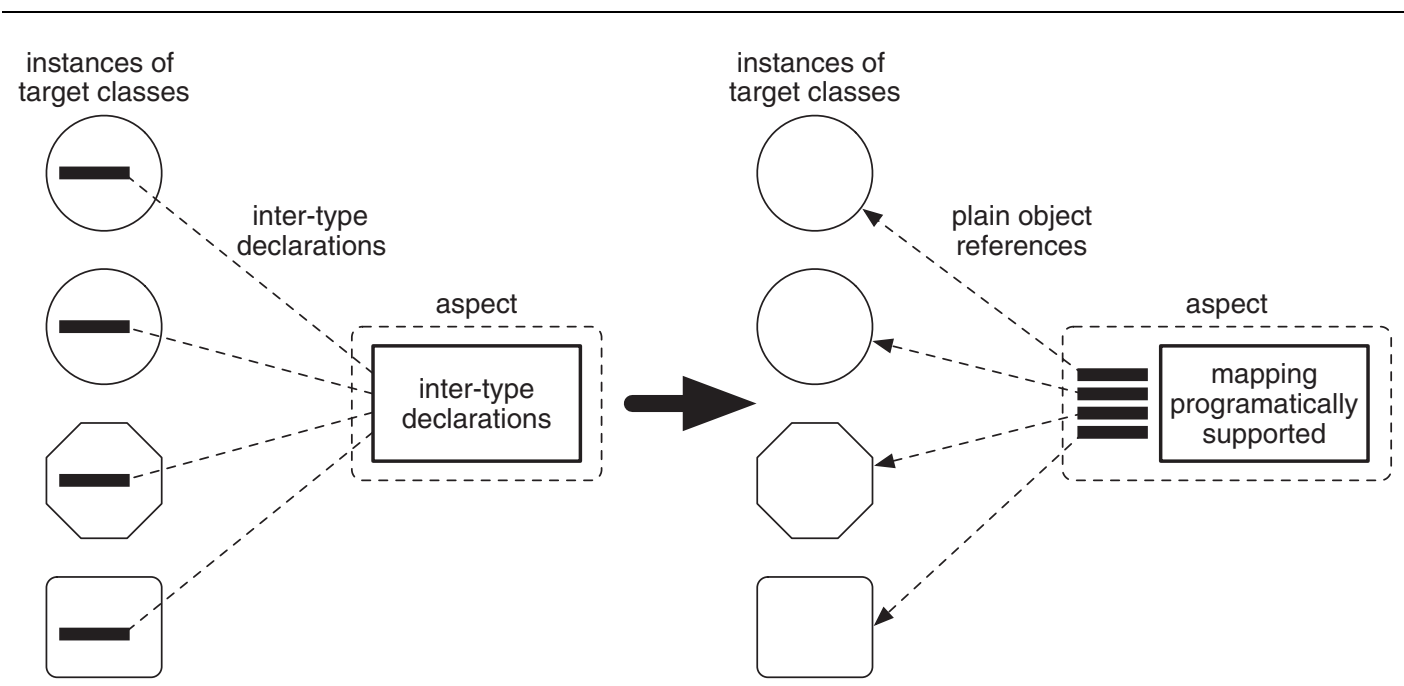

Figure 2. Illustration of the structural effect obtained by the removal of the aspect laziness smell.

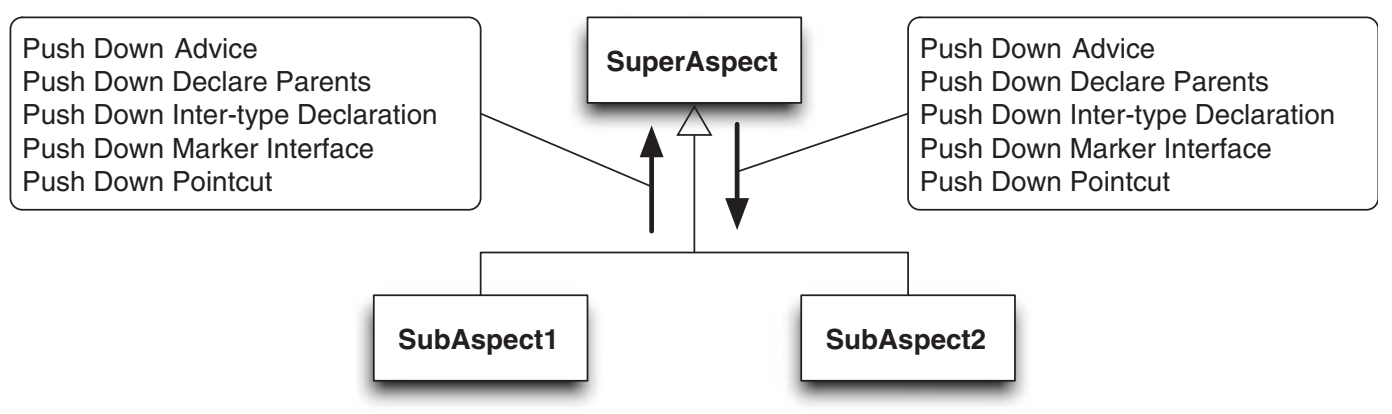

Figure 3. Structural effects performed by the refactorings from Table III.

The second path is described in Section 6. It diverges from the first path from the beginning of the second phase (tidying up). In it, ObserverProtocol is added to the system and therefore Extract Super-aspect (third phase of the first path) is not needed.

It is important to note that the refactoring processes described in this paper correspond to just two of many possible paths. Though the finishing point of the various paths should result in an implementation of the intended design in all cases, it is possible to achieve it through multiple different paths, since there are several possible alternatives to choose from after each step.

Throughout the description of the process, code listings and code fragments are used to illustrate relevant details. In many occasions, changes from the previous illustration are highlighted in bold, following the example from [1]. Likewise, deleted sections of code are signalled in strike-through in some occasions. 
be public and for this reason we make both breakfastTime methods public

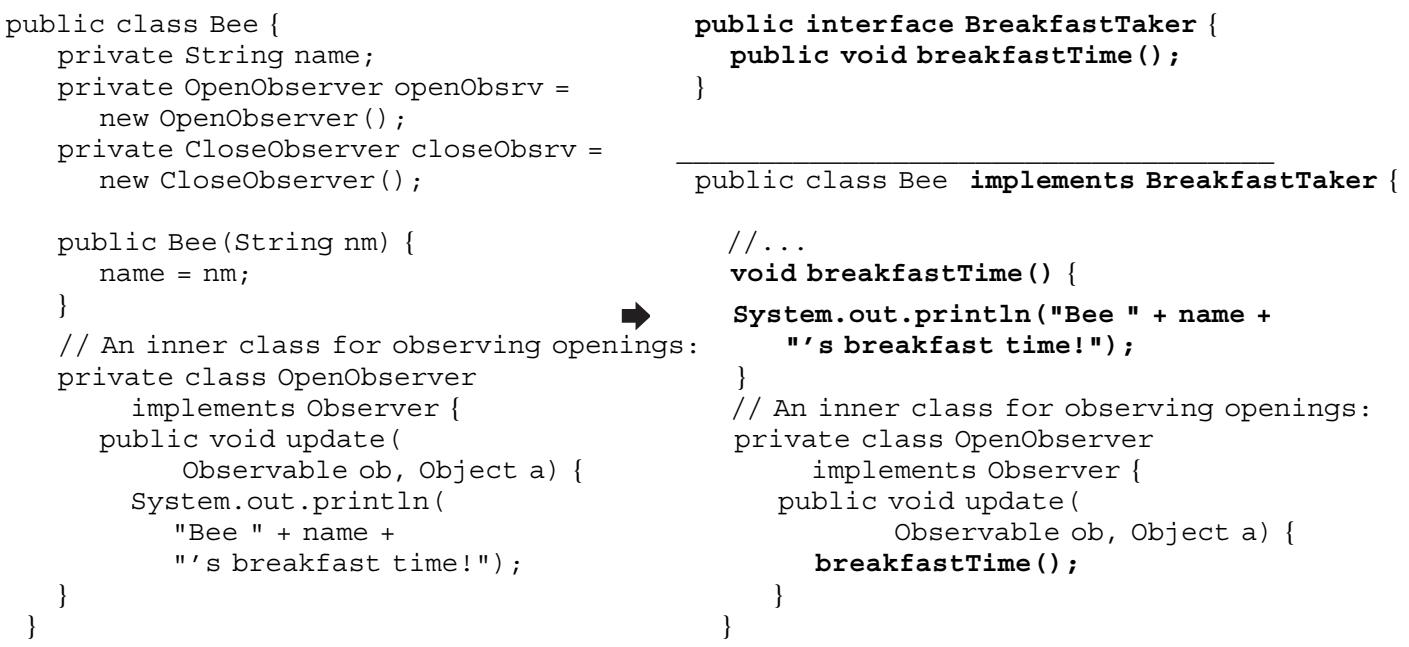

Next, we apply Extract Inner Class into Standalone and use the new interface as the type of the 'former enclosing object'. The code is now ripe for the extraction of the various elements to an aspect. The blank aspect ObservingOpen is created and we apply the following refactorings:

- Move Field from Class to Inter-type to field Flower.oNotify. The private visibility of oNotify is (temporarily) relaxed to package-protected.

- Move Method from Class to Inter-type to method Flower.opening.

- Extract Fragment into Advice to the call to method Flower.oNotify.notifyObservers.

- Extract Fragment into Advice to the call to method Flower.oNotify.close.

The above refactorings move to the aspect all code using field oNotify, so it is now possible to make the field private again, but this time relative to the aspect. Code that initializes the moved fields is moved along with them. The aspect now has the following contents:

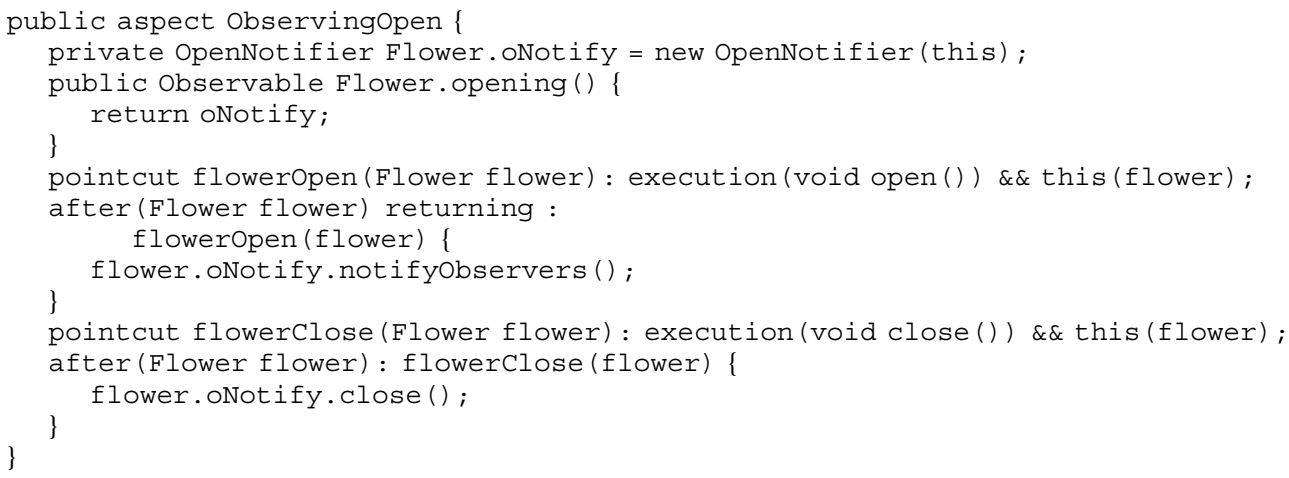

Flower is now clean of code related to the first observing relationship. The next step is to extract from observer classes Bee and Hummingbird all their remaining elements related to the concern. We apply Move Field from Class to Inter-type to Bee.openObsrv. 
Fields are usually private to their owner classes. For this reason, Extract Feature into Aspect recommends that fields be moved before methods that use those fields. That entails relaxing the visibility of the fields, as they are temporarily referred by code from multiple modules. In this case, field visibility is relaxed from private to package protected. The documentation of Move Field from Class to Inter-type recommends that a 'scouter' declare warning be temporarily added to the aspect, to ensure all points referring to the fields are located:

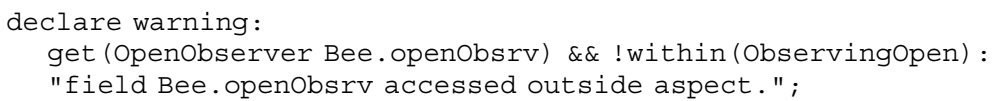

The declare warning signals a use of the field outside the aspect, in method Bee.openObserver. In principle, a method that uses a field related to a concern is likely to belong to that concern. That is indeed the case with Bee.openObserver and therefore we apply Move Method from Class to Inter-type to it. The warnings are gone, so the declare warning is removed and the visibility of field openObsrv is changed back to private (to the aspect). Similar refactorings are applied to Hummingbird. Both observers are now devoid of any code related to the first observing relationship, except for the implements clause referring to BreakfastTaker:

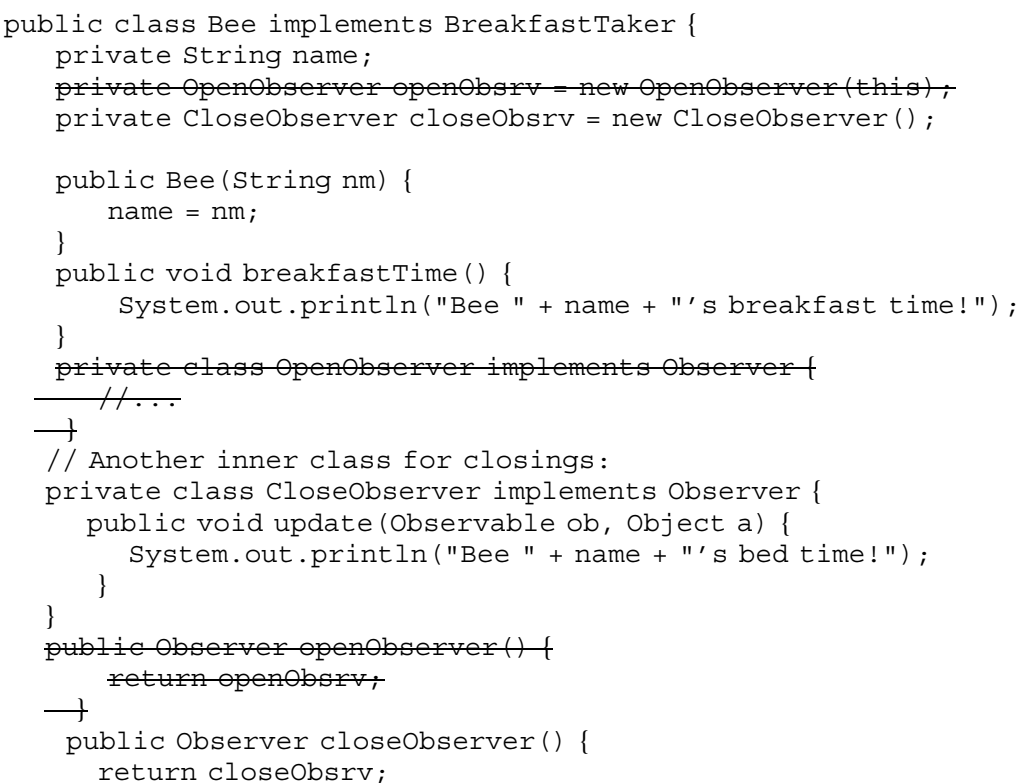

At this point, aspect ObservingOpen contains the following code:

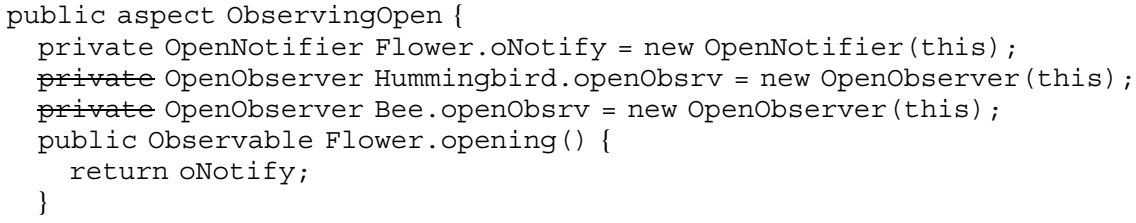




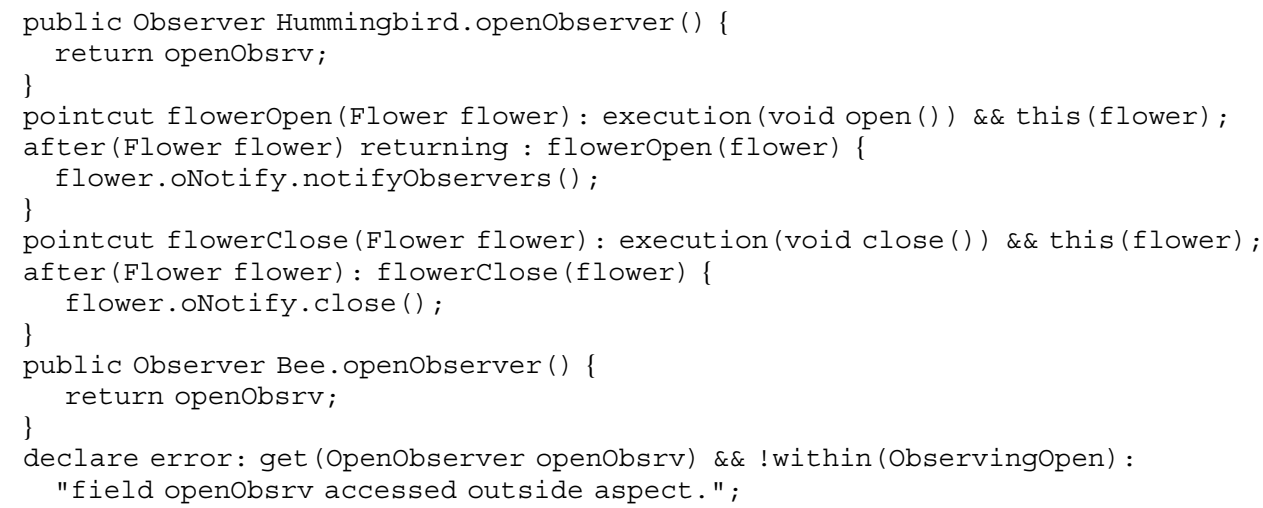

The next task comprises the extraction of the second observing relationship, through a similar sequence of steps. This exposes a significant amount of duplication between the aspects, which warrants further refactorings (see Section 5.3). The second extraction comprises the following steps:

- Apply Extract Inner Class to Standalone to class CloseNotifier within Flower.

- Create a new blank aspect ObservingClose.

- Apply Move Field from Class to Inter-type to field Flower.cNotify, whose visibility is temporarily relaxed from private to package protected. This refactoring entails creating a declare warning exposing three points in Flower that still use the field.

- Apply Move Method From Class to Inter-type to Flower.closing, which removes one warning. The import statements in Flower can now be removed.

- Apply Extract Fragment into Advice to the calls to cNotify.open and cNotify.notifyObservers. This removes the two remaining warnings exposed by the declare warning, so the declare warning is removed and the field Flower.cNotify is made private (to the aspect).

From this point on, Flower is clean of any code related to observing relationships. Next, we deal with the remaining code in the observer participants, Bee and Hummingbird. The first thing to do is to unify both CloseObserver inner classes within Bee and Hummingbird, so that Extract Inner Class into Standalone can be applied to both classes simultaneously, yielding a single stand-alone class. This entails: (1) applying Extract Method [1] to create method bedtimeSleep in each of them; (2) use Extract Interface [1] to extract BedtimeSleeper, just as in the actions that yielded method breakfastTime and interface BreakfastTaker:

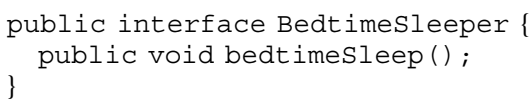

Now, we can use Extract Inner Class into Standalone to both CloseObserver inner classes to yield the common stand-alone class CloseObserver:

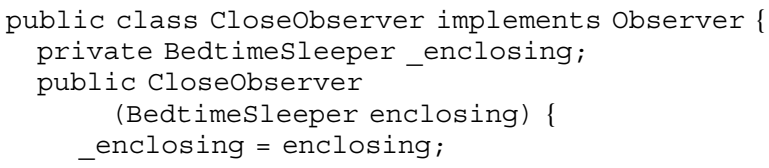




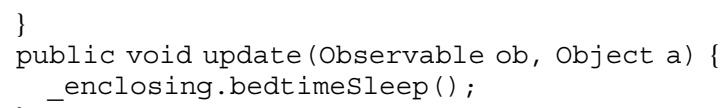

We then move all remaining members related to the extracted concern to the second aspect:

- Apply Move Field From Class to Inter-type to Bee.closeObsrv.

- Apply Move Method From Class to Inter-type to Bee.closeObserver.

- Apply Move Field From Class to Inter-type to Hummingbird.closeObsrv.

- Apply Move Method From Class to Inter-type to Hummingbird.closeObserver.

Some import statements in Bee and Hummingbird can now be removed. The only remaining code in the participants relating to the observing relationships is the implements clauses referring to BreakfastTaker and BedtimeSleeper. We now use Encapsulate Implements with Declare Parents to both Bee and Hummingbird, so that all participants become completely free of any code related to the extracted concerns.

The refactorings performed until now cleaned the participant's code but also created several stand-alone classes and interfaces that provide little functionality and are used only by code placed within the aspects. We therefore inline them so that all codes related to observing relationships are encapsulated in their respective aspects. The former inner classes depend on the interfaces and therefore it is convenient to inline the classes before the interfaces. We apply Inline Class within Aspect to classes OpenObserver, CloseObserver, OpenNotifier and CloseNotifier. Next, we use Inline Interface within Aspect on interfaces BreakfastTaker and BedtimeSleeper. The code related to both concerns is now completely modularized within their respective aspects (Listing 11).

\subsection{Second phase: tidying up the extracted aspects}

At this point, the internal structure of the extracted aspects Listing 11) is confusing and needlessly complex. It contains much duplication and several inner classes and interfaces for whose existence there is no longer a compelling reason. In addition, both aspects betray the Aspect Laziness smell [4]: they statically attach additional state and behaviour to participant classes, while in this case a dynamic and unpluggable composition is advantageous. With the modularization attained through AOP, the scattering effect is just another code smell that can be removed with refactorings [4].

The aim of the refactoring phase described in this section is to improve the internal structure of the aspects, by removing internal duplication and Aspect Laziness, thus bringing the current structure to one closer to current notions of good AO design [13]. These tidying up transformations are prescribed by Tidy Up Internal Aspect Structure, to be used to each aspect in turn. This also makes the internal structure of the aspect more amenable to the subsequent use of Extract Super-aspect, which removes duplication across multiple aspects. We start by refactoring ObservingOpen. As soon as the process is completed, a similar process is carried out on ObservingClose. We first use Generalise Target Type with Marker Interface to eliminate duplication in inter-type declarations resulting from Extract Feature into Aspect. This entails creating inner marker interfaces Subject 


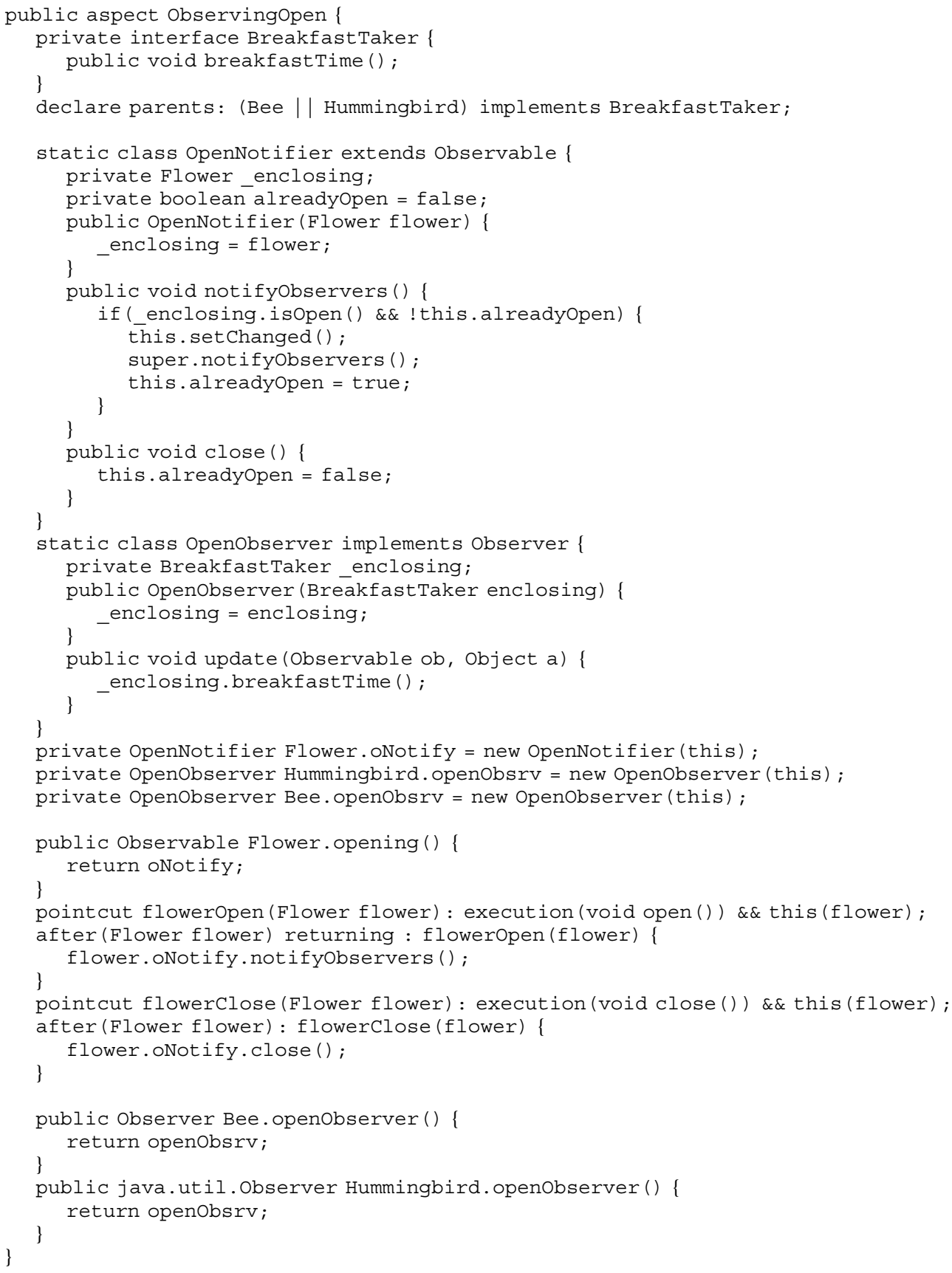

Listing 11. Aspect ObservingOpen just after the extraction of all code related to observing operation open. 
and Observer to represent the pattern roles

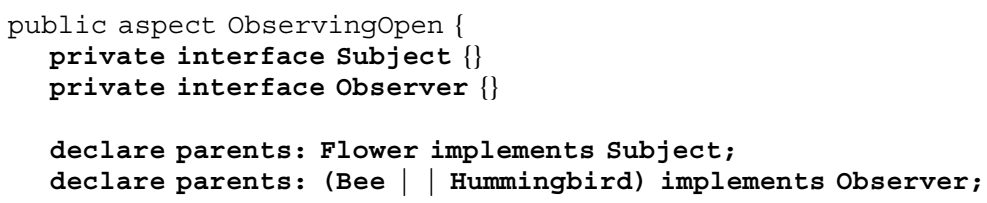

A name conflict arises due to two elements being called Observer. We therefore remove the import statement referring to java.util.Observer and make all references use the full compound name
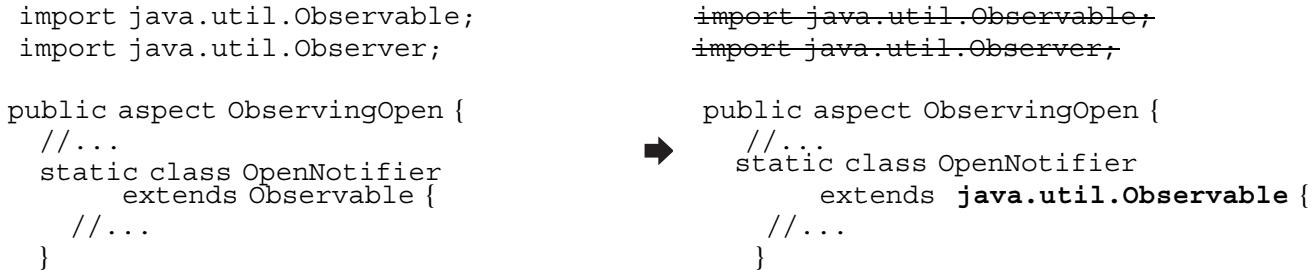

We first apply Generalise Target Type with Marker Interface to type Flower. We do this by replacing all references to Flower with marker interface Subject, including references found within the various OpenNotifier inner classes. This transformation gives rise to a compiler error: the Subject interface does not declare operation isOpen. As a stopgap, we use Extend Marker Interface with Signature on Subject to add the signature of isOpen to Subject. This entails changing the visibility of method Flower.isOpen from package protected to public. Next, we apply Generalise Target Type with Marker Interface to Bee and Hummingbird to eliminate all references to the case-specific interface BreakfastTaker, which are replaced with references to marker interface Observer. Next, interface BreakfastTaker is removed

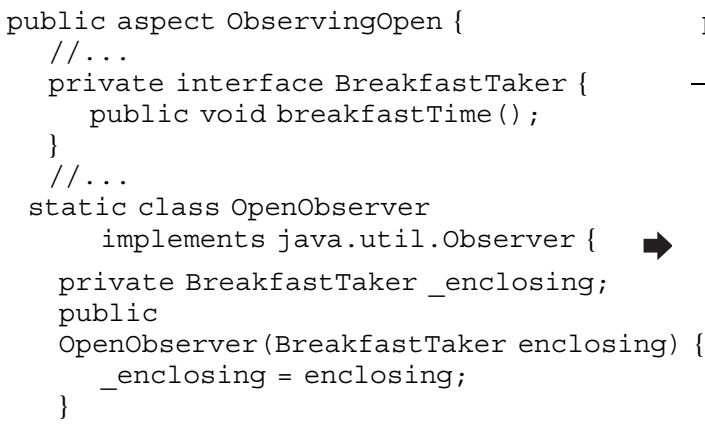

Once more we use Extend Marker Interface with Signature, to add the case-specific signature of method breakfastTime to Observer. This step also eliminates duplication in method openObserver, which is introduced twice (to Bee and Hummingbird). The aspect now refers to the concrete participants only in the declare parents.

Now that some glaring duplication is removed, the next step is to remove Aspect Laziness, by replacing the inter-type state and behaviour with equivalent functionality that is dynamically composable. This entails adding aspect methods akin to the inter-type methods to be replaced and 
then replace the calls to the original inter-type methods in the client code (located within test code from [11], not shown) with calls to the new aspect methods.

What follows is to some extent an elaborated instance of Replace Inter-type Field with Aspect Map. The difference is that in the present case we deal with inner classes rather than inter-type fields. As prescribed in the description of Replace Inter-type Field with Aspect Map, we use Replace Inter-type Method with Aspect Method as a follow-up (the strategy is first to deal with fields and next with the methods that use those fields). The aim of this sequence of refactorings is to perform the transformation sketched in Figure 3. They add a mapping structure to the aspect, along with the associated logic. Note that the new logic includes one operation (clearObservers) that is not present in the abstract aspect presented in [13]

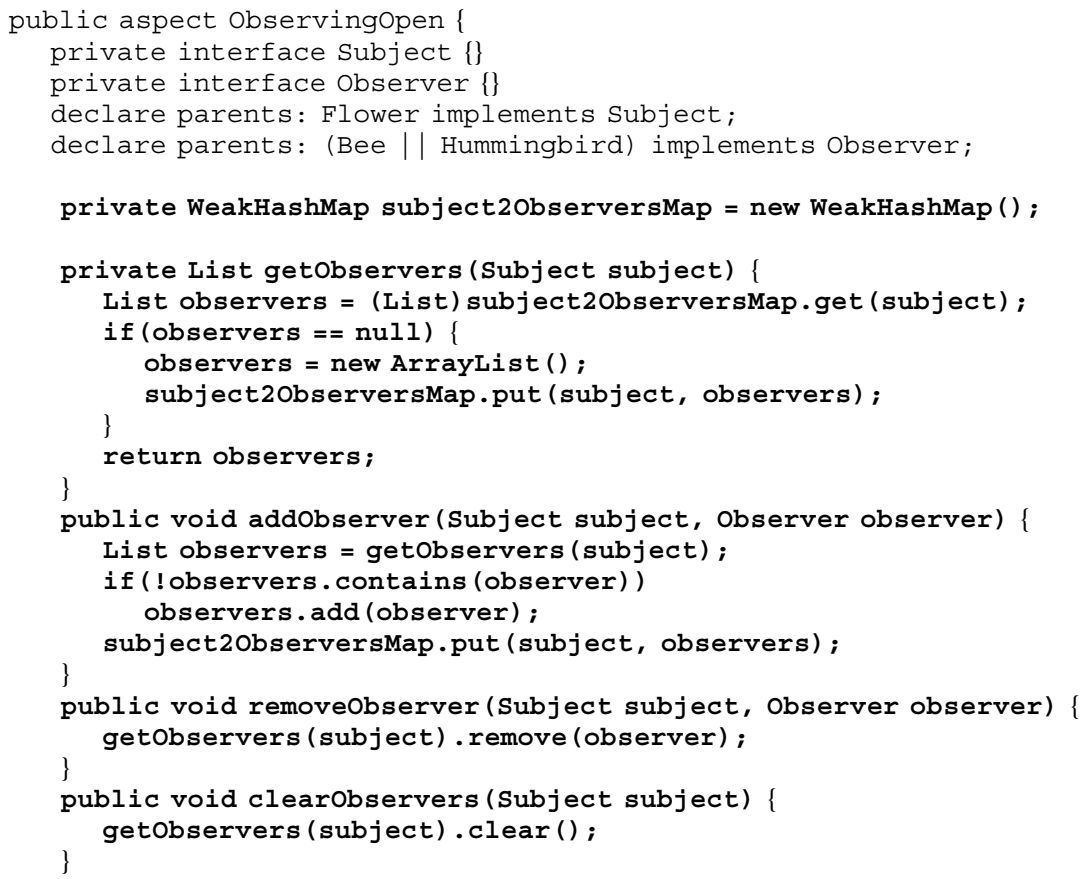

Next, Replace Inter-type Method with Aspect Method is used, which entails adding aspect method notifyObservers. The method provides the same functionality as OpenNotifier.notifyObservers, using a boolean field (alreadyOpen) newly added to Subject, to be used for the same purposes as field alreadyOpen of inner class OpenNotifier:

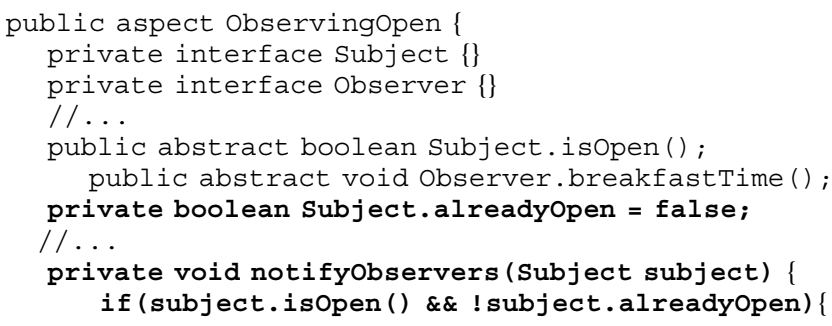




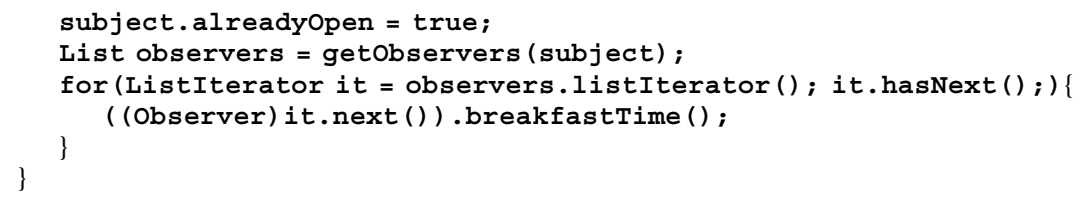

Also as prescribed in Replace Inter-type Method with Aspect Method, we add a declare warning to expose all places where the logic to be replaced is located. The declare warning targets method Subject.opening, the accessor method for the instance of inner class OpenNotifier

declare warning: call (java.util.observable opening()) : "opening() called here." ;

Compiling after the declare warning is added exposes six warnings, all pointing to client (unit test $^{\dagger \dagger}$ ) code. We replace the original calls with calls to aspect logic. One example is as shown:

f.opening () .addobserver (ha.openobserver());

Observingopen. aspectof () . addobserver ( $f$, ha) ;

Tidying up the second aspect: Improving the internal structure of ObservingClose requires essentially the same steps as with ObservingOpen, i.e. another instance of Tidy Up Internal Aspect Structure. The steps are:

- Removal of imports of java.util.Observable and java.util.Observer. The compound names are used instead.

- Creation of private inner interfaces Observer and Subject as a preliminary step to using Generalise Target Type with Marker Interface.

- Applying Generalise Target with Marker Interface to Flower: references to Flower are replaced by references to marker interface Subject.

- While using Generalise Target with Marker Interface, Extend Marker Interface with Signature is used to introduce method isOpen to Subject. References to Bee and Hummingbird are replaced by references to marker interface Observer. Interface BedtimeSleeper is removed (along with a declare parents that served to add a signature). Extend Marker Interface with Signature is used once more to extend type Observer with the signature of bedtimeSleep.

- Use of Replace Inter-type Field with Aspect Map, followed by Replace Inter-type Method with Aspect Method to add a new implementation to ObservingClose. As prescribed by Replace Inter-type Method with Aspect Method, a declare warning is added to expose calls to accessor method CloseNotifier.closing:

declare warning: call(java.util.observable closing()) : "closing() called here." ;

- Following the locations exposed by the declare warning, the calls to CloseNotifier.closing (in test code, not shown) are replaced. Again, we must reverse the order in which observers are registered. We remove the declare warning, compile and the test passes.

\footnotetext{
${ }^{\dagger}$ In the course of the transformations described, an assertion within test code fails at a given point, due to two implementations traversing in opposite orders the list of observers to be notified. The order of notification of observers is not relevant but the particular way with which Eckel's original test was adapted needlessly hard coded the original order of notifications. For this reason, the order with which observers are registered in the test is inverted.
} 


\subsection{Third phase: factoring out common code to a super-aspect}

Though the refactored aspects are better formed, when considered together they betray much duplication across aspects, namely in the marker interfaces, field subject2ObserversMap and associated logic. We eliminate the duplication by using Extract Super-aspect to factor out common code to a super-aspect. This entails performing the following steps:

- Create blank abstract aspect ObservingRelationships.

- Aspects ObservingOpen and ObservingClose are made to extend ObservingRelationships.

- Use Pull Up Marker Interface on inner interfaces Subject and Observer of both aspects, to move them to ObservingRelationships. Their visibility is relaxed from private to protected.

- Use Pull Up Field [1] on the field subject2ObserversMap of both aspects.

- Use Pull Up Method [1] on methods getObservers, addObserver, removeObserver and clearObservers of both aspects.

Method notifyObservers is another candidate to being pulled up, but it depends on too many casespecific members. For this reason, we merely extract to the super-aspect its abstract declaration. Pointcuts flowerOpen and flowerClose are also case specific and for this reason we refrain from adding more abstract declarations to the super-aspect. This decision illustrates one of the advantages of refactoring: as the code can be changed in the future, design decisions do not have to be made upfront. Developers have the option to change their minds at a later phase, and refactor. The extracted super-aspect (very similar to ObserverProtocol from [13]; Listing 9) is shown in Listing 12 and ObservingOpen is shown in Listing 13.

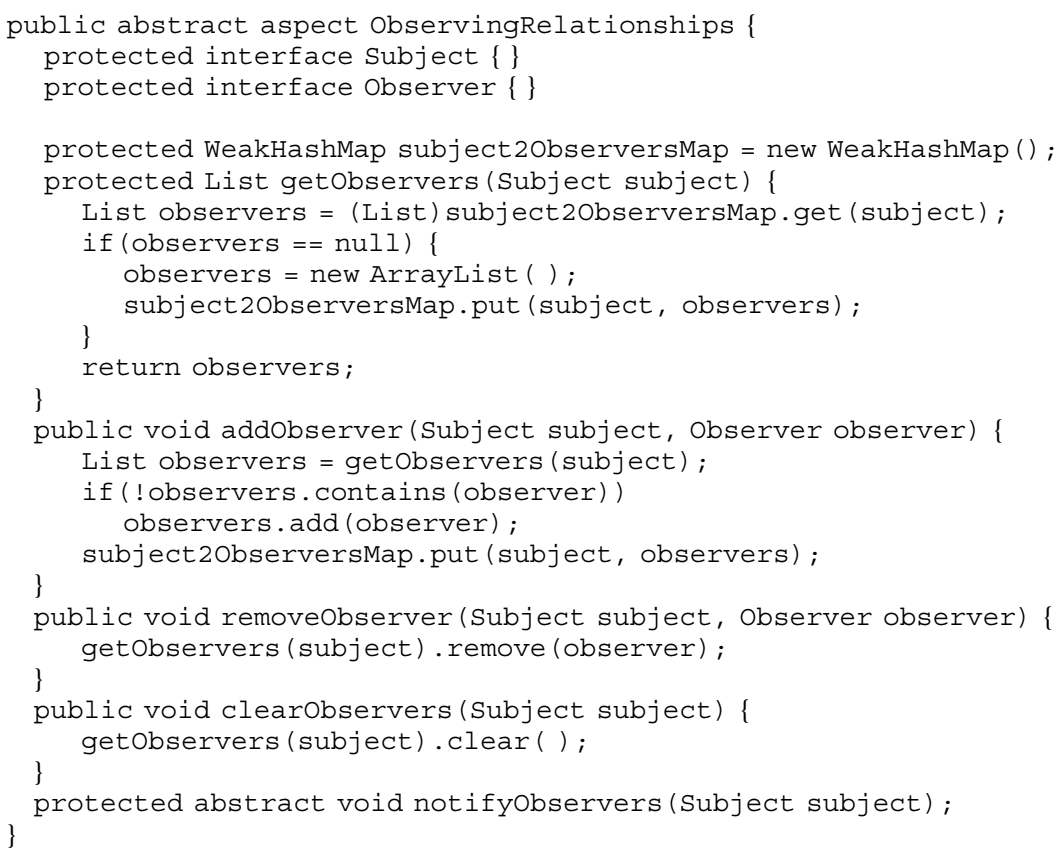

Listing 12. Stable form of the ObservingRelationships abstract aspect. 


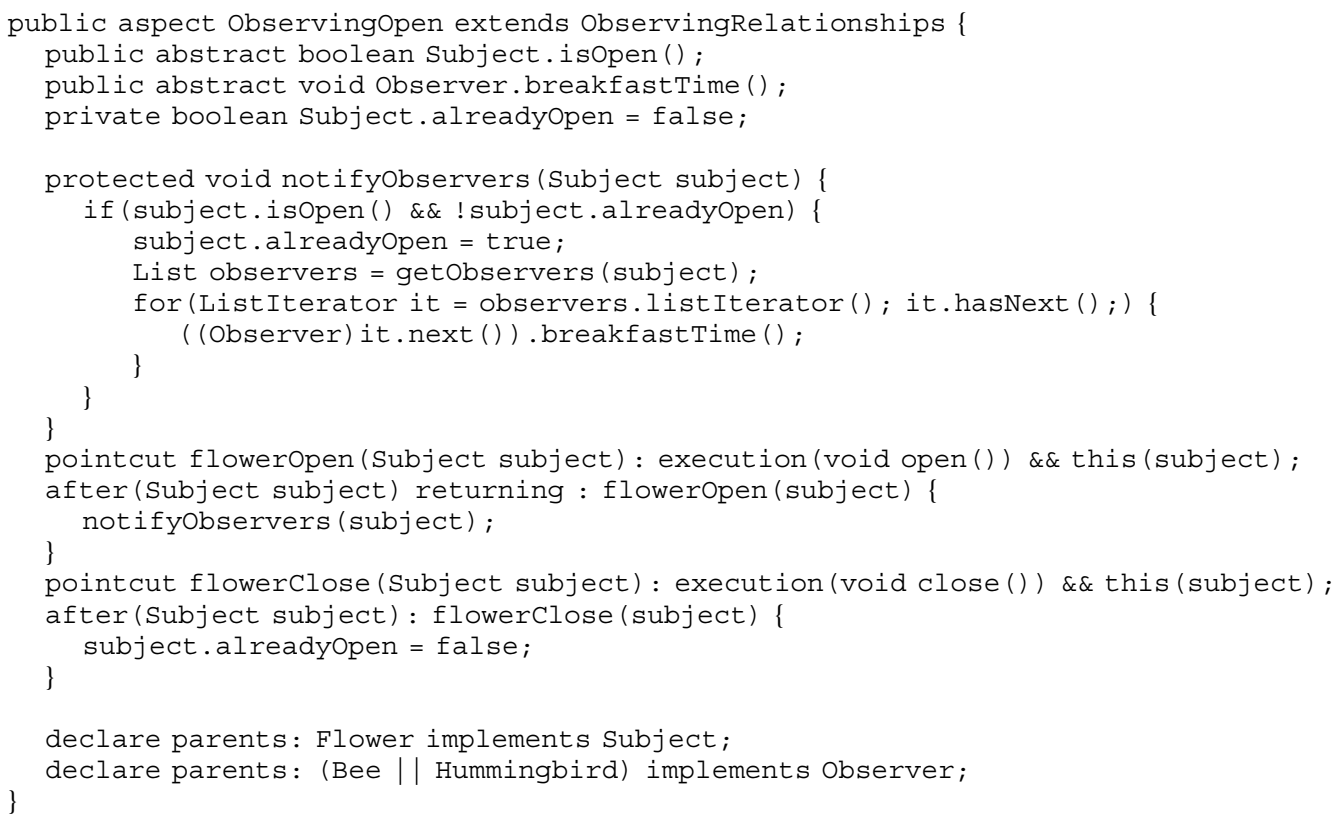

Listing 13. ObservingOpen after factoring out duplicated code to the super-aspect.

\section{ALTERNATIVE REFACTORING PATH}

The previous section shows how to use refactoring to gradually transform a Java implementation of Observer into an implementation in AspectJ. However, we also have the option of reusing the ObserverProtocol aspect from [13]. It is equally feasible to take advantage of this ready-made aspect while refactoring the case-specific aspects of the example. In this section, we describe such an alternative refactoring path.

The alternative path starts diverging from the one described in the previous section after the completion of the extraction process (Section 5.1). It almost exclusively involves adding just the case-specific parts, as ObserverProtocol already provides the most of the generally applicable parts. The existing structure must be tidied up as previously, but because ObserverProtocol is included, the alternative path involves adding fewer amounts of new code compared to the previous path.

Again, we tackle one aspect at a time, starting with the use of Tidy Up Internal Aspect Structure on ObservingOpen. This refactoring prescribes the use of Generalise Target Type with Marker Interface, which requires adding marker interfaces Subject and Observer representing the participant roles in the pattern. This time we do not need to add them as they are declared in ObserverProtocol. Instead, we make ObservingOpen inherit from ObserverProtocol. This in turn gives rise to several issues. One is the conflict caused by two member types being named Observer, which we again solve by removing the import to java.util.Observer and making all references to the interface use the full compound name. Another issue is the need to provide 
of Observer) only compiles because all references to BreakfastTaker were first replaced with Observer
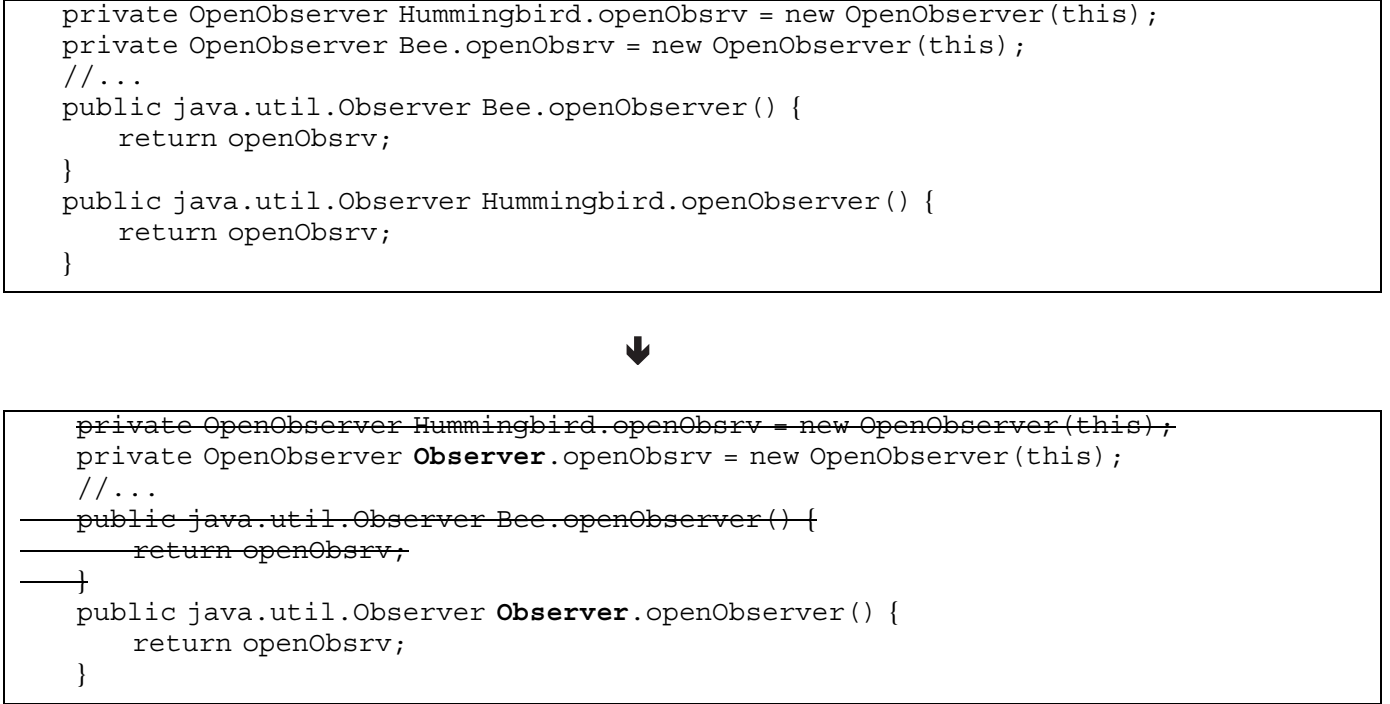

This completes the application of Generalise Target Type with Marker Interface to types Hummingbird, Bee and Flower. From this point on, participants are referred only in the roleassigning declare parents clause. In the process, pointcut flowerOpen is removed as well, as it is identical to subjectChange. Further improvements on the internal structure of ObservingOpen require the replacement of the current implementation with the one defined in ObserverProtocol. Only then it is possible to remove the inner classes and the dependence on the Observable/Observer protocol from java.util.

There are a few hurdles. ObserverProtocol expects the events triggering the reactions of the observers to be represented by a single pointcut-subjectChange-but in this particular case, it is convenient to use two, to account for the two different operations of Flower. In addition, this case requires that notification of all observers in the subject's list depend on the result of a test (if it is the first occurrence of a sequence). Only if the test succeeds are the subject's registered observers notified. This test relies on boolean field alreadyOpen of inner class OpenNotifier. The field should be moved to Flower, as an inter-type declaration, but the point where the test is made lies within the abstract base aspect ObserverProtocol, in a piece of advice acting on the joinpoints captured by pointcut subjectChange:

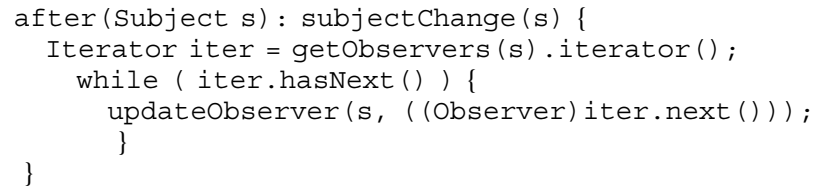

Therefore, ObserverProtocol must incur invasive changes to be used in this example. A subaspect cannot override advice inherited from the super-aspect and therefore the advice must be 
pushed down from ObserverProtocol to ObservingOpen, through Push Down Advice. In addition, functionality is missing in ObserverProtocol: the ability to clear all observers subscribing to a given subject. We therefore add the method clearObservers (identical to the one added during the tidying up phase described in Section 5.2) to ObserverProtocol.

ObservingOpen now has two pieces of after advice acting on this pointcut, related to the old and new implementations, respectively. Two advice of the same kind acting on the same pointcut within a single aspect clearly seems bad style_-in the normal case, the two advice blocks should merge into a single advice, perhaps with calls to suitably named auxiliary methods. However, the advice related to the original implementation is about to be removed. We now add the missing logic to ObservingOpen. It is based on the alreadyOpen field introduced to Subject. This involves adapting the advice pulled down from ObserverProtocol according to the rules of this case:

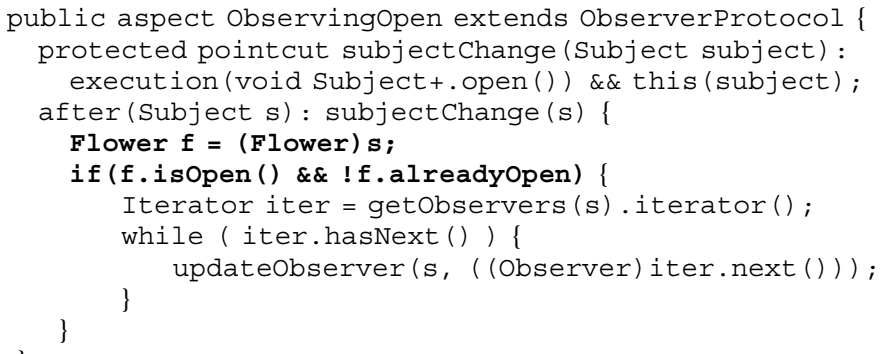

We add the advice acting on the pointcut that captures the execution of method Flower.close:

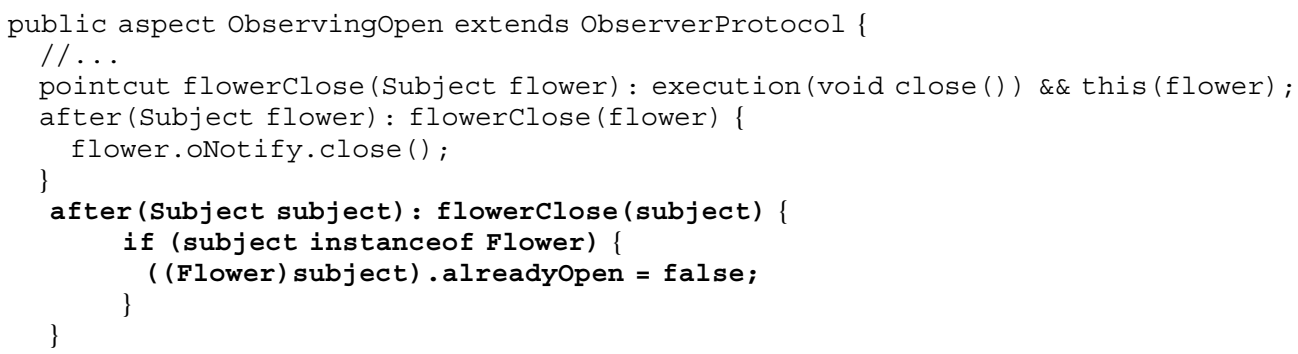

Finally, we provide aspect method updateObserver with the logic suitable for the new implementation:

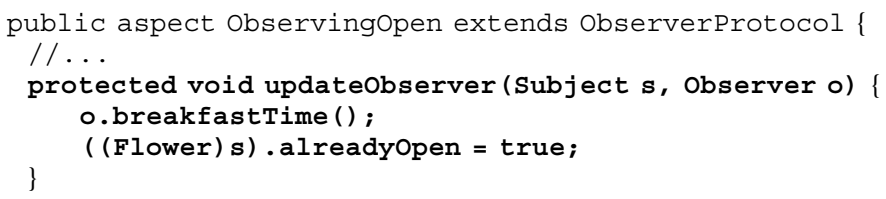

We now remove the original implementation. We could consider one last tidying up to do, related to the breakfastTime signature that is still extending interface Observer. Though we could replace 


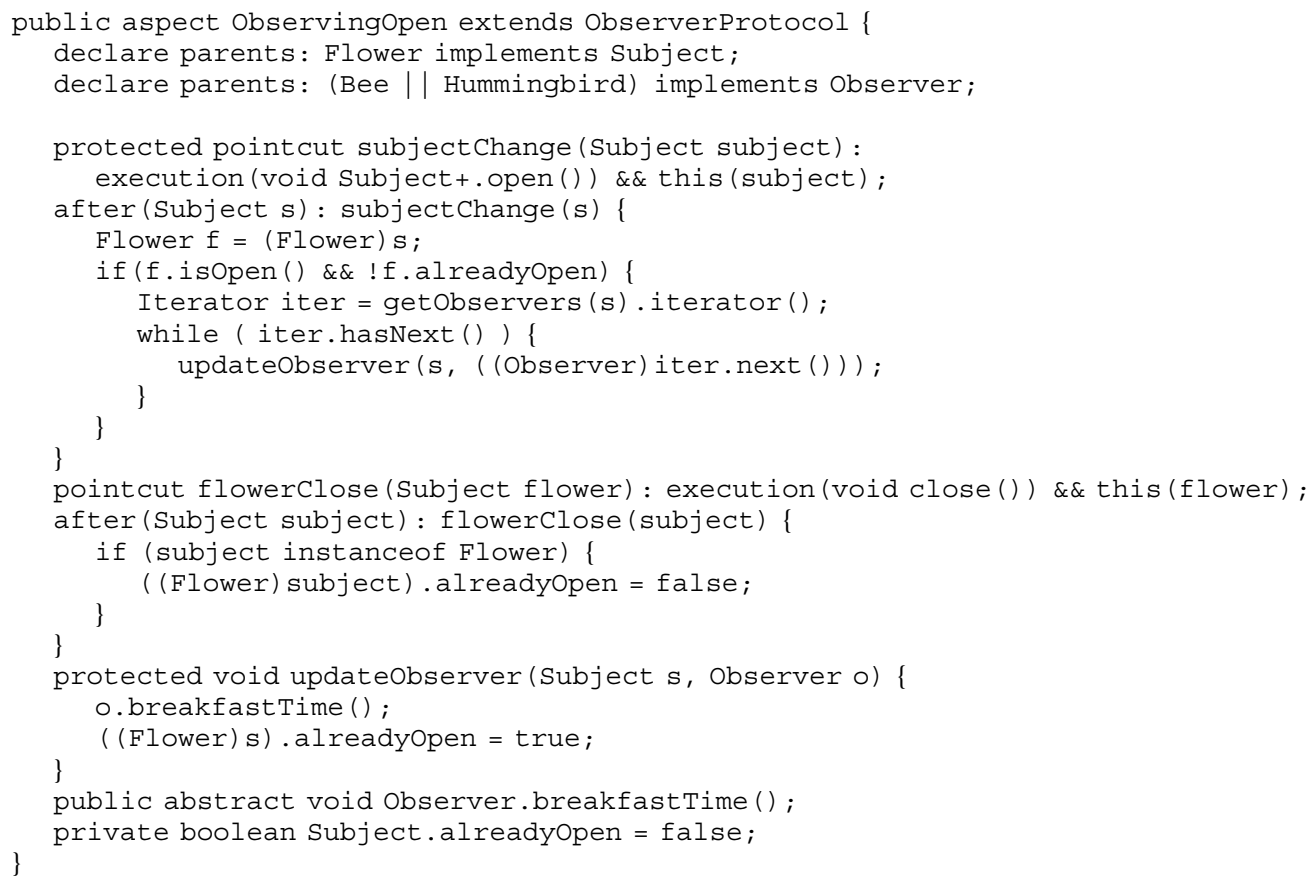

Listing 14. Final version of ObservingOpen obtained from the alternative path.

it with type conversions in the points where breakfastTime is used, it is proving rather convenient because it is affecting two different and unrelated types, thus avoiding duplication. We choose to leave it there for now. Listing 14 shows the final version of ObservingOpen. We now perform a similar sequence of steps to the second aspect, yielding similar results. This time there is no need to make more methods public.

\section{DISCUSSION}

We base our work on the hypothesis that evolving a complex collection of scattered code fragments in systems of realistic dimensions is a costly and risky process and that the modularization of such collections, made possible by AOP, bring benefits to comprehensibility and evolution [33]. We assume that the larger is the system, the larger is the expected benefit to comprehensibility and ease of evolution that modularization can bring.

Hannemann [13] analyses the GoF patterns and their AspectJ implementations according to the roles defined by the pattern. The pattern roles are classified as superimposed when the object has a distinct, primary role besides the one assigned by the pattern. Otherwise, pattern roles are classified are defining, i.e. the object exists only to play the role in the pattern. Such roles do not lend themselves for a separation of roles because the participant plays only that role. The authors acknowledge that role classification into superimposed and defining is not clear-cut in some cases. Classes with superimposed roles betray the Double Personality smell as we define it in [4,9]. 
Conceptually, superimposed roles can be plugged and unplugged from existing objects. However, OO languages are often unable to do that, because they do not modularize superimposed roles such as those defined by Observer. Instead, code related to the roles is scattered throughout participant classes. In terms of reusability and ease of evolution, it seems reasonable to assume that patterns defining superimposed roles will derive significant benefits from AOP. Among the GoF patterns, the benefits brought by Observer are particularly noticeable, as it is the only pattern that includes two superimposed roles, in addition to not including any defining roles. Consequently, the AspectJ implementation of Observer modularizes a whole collaboration of objects. That is one reason why in this case we observe a significant improvement over traditional OO.

Experience gained from using Extract Feature into Aspect shows that extractions of class members based on inter-type declarations do not change the original design, they merely isolate its various elements in a single module at the source code level. This makes the internal structure of the extracted aspect awkward to deal with and may betray the Aspect Laziness smell [4]. Such modules can benefit from changes to its internal structure, if not a downright replacement of the original internal design and implementation. That provides the motivation for using Tidy Up Internal Aspect Structure and modularization is a prerequisite for applying it.

The refactoring process described in this paper also suggests that it is hard to derive reusable modules, even with AOP. Though the abstract aspect from [13] is potentially reusable, it had to undergo invasive changes in order to adapt it to the simple Java example by Eckel [11].

As regards the obliviousness property [32], participant classes do become oblivious to roles defined by the pattern after the system is refactored to AspectJ. However, it is still necessary for some part of the system to remain aware of the fact that participant classes play the additional roles, in order to set relationships and perform configurations. In Eckel's example, that role is played by test code (not shown in the paper); in the example by Hannemann and Kiczales [13], it is played by a main method. Those parts are responsible for calling aspect methods to register and unregister observers to a given subject. Conceptually, such parts reside at a different level than participant classes and it seems reasonable to expect them not to be oblivious to the aspects.

It is important to note that the definition of obliviousness proposed in [32] applies not only to a set of classes but also to the programmers that develop the classes. We do not abide by that more demanding view of obliviousness. Although classes do not betray code that is dependent on aspects throughout the process, the code base must be refactored in order for it to expose the joinpoint leverage that aspects need. One example is the isOpen method, which was extracted from the class playing the role of Subject (see Section 5.1). Our experiments suggest that although class obliviousness is desirable and often achievable, programmer obliviousness raises important issues related to the development process and does not seem to be feasible in practice. We give the name aspect-friendly [3] to a code base that is refactored to provide the necessary leverage to aspects but remains code oblivious to any specific aspect code (i.e. a system build can be performed without including the aspects in it).

The experience gained from deriving the refactoring process described in this paper, as well as other experiments [9], suggests that manual extraction of CCCs in systems of realistic dimensions involves a significant amount of work. For this reason, we think it is highly desirable that automated support [34] be provided in tools and developing environments. Future work includes testing the refactorings illustrated in the paper in larger and more complex systems. 


\section{RELATED WORK}

The refactoring process described in this paper comprises a first use and illustrating example of a collection of novel refactorings for AOP code that we describe in [3,4]. In [4], we propose a few novel code smells, including Double Personality and Aspect Laziness. Several other authors report on the successful reuse of those refactorings. van Deursen et al. [36] describe the analysis of the JHotDraw framework ${ }^{\ddagger \ddagger}$ and the extraction of several CCCs from its code base. They report using Encapsulate Implements with Declare Parents and Move Method from Class to Inter-type. Fuentes et al. [37] report on refactoring the Java code base of an ambient intelligence application and mention benefits derived from our refactorings [4,9]. Likewise, Kulesza et al. [38] report on refactoring the JUnit framework ${ }^{\S \S}$ mainly on the basis of those refactorings.

Hannemann [13] presents implementations of the 23 GoF patterns [10] in both Java and AspectJ. They were able to modularize the implementation of 17 of the patterns, 12 of which had part of the implementations abstracted into reusable aspect modules. In a few cases multiple instances of the aspect can be transparently composed into a system. Observer is one of the patterns, whose design is used as the target of the refactoring processes described in this paper. The authors present an analysis of the two sets of implementations and conclude that improvements of the AspectJ implementations over the Java ones are directly correlated to the presence of crosscutting structure in the patterns. The crosscutting effect arises in patterns that superimpose additional (secondary) roles on participant classes and whose implementation code cuts across participant classes.

Hannemann et al. [34] and again Hannemann [35] propose that refactoring support for AOP be divided into three categories: aspect-aware $O O$ refactorings (a concept previously proposed by Hanenberg et al. [5]), aspect-oriented refactorings (i.e. refactorings that specifically target AO constructs, such as those used here) and refactorings of CCCs, i.e. refactorings in which the scattered elements comprising a target CCC and their individual transformations are considered together, instead of handling each element separately. It does not seem possible to carry out, as a single operation, the latter category of refactorings without the support of a tool and the focus of [34] is to present one such tool. However, a sequence of AO refactorings, such as the process described in this paper, achieves a similar effect as a refactoring of CCCs as understood by Hannemann.

Like us, Hannemann et al. [34] use Observer as a basis for an illustrating example of a refactoring process that results in the modularization of an implementation of Observer. They provide an outline of the steps to be carried out, but it is significantly less detailed than the one we present in this paper. The outcome of their illustrating refactoring is the Aspect J design for Observer presented in [13], which we also use in this paper. As it would be expected, there are similarities between the transformations presented in the outline and the ones we describe here. However, more details than those provided in [34] are required for a thorough comparison and analysis.

Several other authors use the GoF patterns, again with an emphasis on Observer, to illustrate the relative advantages of AOP [4,39-41]. The comparison presented in [40] is interesting in that the comparison made is not between $\mathrm{OO}$ and $\mathrm{AO}$ implementations of Observer, but between the AspectJ implementation proposed in [13] and a different AO implementation coded in Caesar.

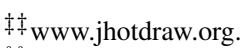

$\S \S$ www.junit.org. 
The comparison stresses the disadvantages of the AspectJ implementation relative to the Caesar implementation as regards internal modularity, polymorphism and compositional capabilities.

Iwamoto and Zhao [6] announce their intention to build a catalogue of AOP refactorings. They present a catalogue of 24 refactorings, but the information provided about them is limited to the names of the refactorings. In this paper, we provide more detail about the AO refactorings used throughout the process described in this paper, even if we refer the full documentation to [3,4,9].

Hanenberg et al. [5] also propose three AOP refactorings-Extract Advice, Extract Introduction and Separate Pointcut. Their proposed Extract Advice corresponds to our Extract Fragment into Advice and their proposed Extract Introduction corresponds to our Move Field from Class to Intertype and Move Method from Class to Inter-type. Separate Pointcut relates to evolution of pointcuts and has no correspondence in our collection. The latter refactoring concerns good style for pointcuts, expressing the notions that pointcuts should in most situations be named rather than anonymous, and be as decomposed as possible to enhance their reusability. The latter notion is also argued by Lagaisse and Joosen [42]. Separate Pointcut is not needed in the process described in this paper because anonymous pointcuts are avoided. In addition, the declaration and use of pointcuts is in part constrained by the existing interface of ObserverProtocol.

Laddad [7] presents a collection of refactorings tailored to J2EE applications, covering a different area of the AO refactoring space than the refactorings used in the process described in this paper. The refactorings vary widely in both level and scope of applicability, including generally applicable refactorings like Extract Interface Implementation, Extract Method Calls and Replace Override with Advice, but also concern-specific refactorings such as Extract Concurrency Control and Extract Contract Enforcemement. Some refactorings target specific AO design patterns [28], e.g. Extract Worker Object Creation and Replace Argument Trickle by Wormhole and Extract Exception Handling. Some of the refactorings are presented with only a mention to the name and a brief motivating paragraph. van Deursen et al. [36] report on using Extract Method Calls. Kellens and Gybels [43] provide a description of Extract Method Calls that is more detailed and analyse the refactoring in the light of its automated application.

van Deursen et al. [36] propose a few new refactorings based on their work on JHotDraw, though without providing much detail. These include Move Role to Aspect, which entails moving code related to a secondary role to an aspect. This is actually a refactoring that removes Double Personality. The refactorings we propose in [4] are more low level but can achieve similar results through a suitable sequence. van Deursen et al. also propose Move Observer to Aspect, a more high level compound refactoring that moves an entire implementation of Observer to an aspect. In addition, the authors propose Override Method with Advice for Overlapping Roles, which applies to methods that perform multiple features relating to multiple roles. The refactoring extracts a method definition to the Java interface to which the method belongs. An aspect uses an around advice to override the default behaviour provided by the extracted method in the situations where it is suitable. Finally, the authors propose Advise Method Overrides, a refactoring that extracts to an aspect the idiom comprising duplicated statements common to the (start or end of) all method overrides of a given (superclass) method.

There are a number of works describing experiments in refactoring existing OO code bases, with various degrees of detail provided. In [3], we report on our experiments in extracting a concern from a Java framework for workflow applications. Tonella and Ceccato [44] report on the results obtained in extracting the implementation of interfaces (approached as symptoms of latent aspects) from the source code of some of the packages in the standard Java library. Bruntink et al. [45] 
report on the refactoring of specific concern-parameter checking-to separate modules coded in a domain-specific language. Colyer and Clement [46] describe an experiment in refactoring a middleware product line with tens of thousand classes, many millions of lines of code and hundreds of developers. Zhang and Jacobsen [47] describe the refactoring they performed of ORBacus, an industrial strength CORBA implementation.

Cole and Borba [48] propose programming laws from which refactorings for AspectJ can be derived. The authors focus on the use of their laws to derive existing refactorings such as those proposed in $[3,4]$, and describe two case studies in which the laws were tested, comprising the extraction of concurrency control and distribution, respectively. Most of the proposed laws relate to the extraction of CCCs to aspects, and therefore there is some overlap between the refactorings they derive and the extraction refactorings used in Section 5.1. However, their focus is on providing proofs that the transformations are behaviour preserving, while we focus on developing a notion of style for AOP, by increasing its refactoring space. Cole and Borba remark that the extraction procedure for the second case study is generalizable, as the implementation of distribution is commonly used. The authors claim that it is possible to derive a concern-specific Extract Distribution refactoring, though details are not provided.

\section{CONCLUSION}

This paper presents a practical example of a refactoring process that includes the extraction of CCCs to aspects, the subsequent internal restructuring of the extracted aspects and the factoring out of common code to super-aspects. The process described serves as an introduction to the collection of refactorings documented in [4,9], playing a similar role to chapter 1 of [1]. It also complements the various code examples found in [4]. Though the paper was written to stand on its own, it refers to an eclipse project available as an online supplement, containing over 30 complete code snapshots. After the description of the process, we provide a discussion and a survey of related work.

\section{ACKNOWLEDGEMENTS}

This work was supported by FCT (the Portuguese Foundation for Science and Technology) and FEDER (the European Regional Development Fund) under contract SOFTAS (POSC/EIA/60189/2004).

\section{REFERENCES}

1. Fowler M, Beck K, Opdyke W, Roberts D. Refactoring-Improving the Design of Existing Code. Addison-Wesley: Reading, MA, 1999.

2. Kiczales G, Lamping J, Mendhekar A, Maeda C, Lopes C, Loingtier J, Irwin J. Aspect-oriented programming. Proceedings of the 11th European Conference on Object-oriented Programming, Jyväskylä, Finland, 1997 (Lecture Notes in Computer Science, vol. 1241), Aksit M, Matsuoka S (eds.). Springer: Berlin, Germany, 1997; 220-242.

3. Monteiro MP, Fernandes JM. Object-to-aspect refactorings for feature extraction. Industry track paper at the $3 r d$ International Conference on Aspect-oriented Software Development, Boston, MA, 2004.

4. Monteiro MP, Fernandes JM. Towards a catalogue of aspect-oriented refactorings. Proceedings of the 4th International Conference on Aspect-oriented Software Development, Chicago, IL, 2005. ACM Press: New York, NY, 2005; 111-122. DOI: $10.1145 / 1052898.1052908$. 
5. Hanenberg S, Oberschulte C, Unland R. Refactoring of aspect-oriented software. Fourth Annual International Conference on Object-oriented and Internet-based Technologies, Concepts, and Applications for a Networked World (Net.ObjectDays), Thuringia, Germany, 2003. Springer: Berlin, Germany, 2003; 19-35.

6. Iwamoto M, Zhao J. Refactoring aspect-oriented programs. Fourth AOSD Modeling with UML Workshop at UML 2003, San Francisco, CA, 2003.

7. Laddad R. Aspect-oriented Refactoring, Parts 1 and 2, The Server Side, 2003. http://www.theserverside.com/tt/articles/ article.tss?l=AspectOrientedRefactoringPart1, http://www.theserverside.com/tt/articles/article.tss?l=AspectOrientedRefactoringPart2 [25 April 2007].

8. Kiczales G, Hilsdale E, Hugunin J, Kersten M, Palm J, Griswold WG. An overview of AspectJ. Proceedings of the 15th European Conference on Object-oriented Programming, Budapest, Hungary, 2001 (Lecture Notes in Computer Science, vol. 2072), Knudsen JL (ed.). Springer: Berlin, Germany, 2001; 327-335.

9. Monteiro MP. Refactorings to evolve object-oriented systems with aspect-oriented concepts. PhD Thesis, Universidade do Minho, Braga, Portugal, 2005.

10. Gamma E, Helm R, Johnson R, Vlissides J. Design Patterns, Elements of Reusable Object-oriented Software. AddisonWesley: Reading, MA, 1995.

11. Eckel B. Thinking in Patterns, revision 0.9. Book in progress, available online. http://www.pythoncriticalmass.com/downloads/TIPatterns-0.9.zip [16 June 2007].

12. Cooper J. Java Design Patterns: A Tutorial. Addison-Wesley: Reading, MA, 2000.

13. Hannemann J, Kiczales G. Design pattern implementation in Java and AspectJ. Proceedings of the 17th ACM SIGPLAN Conference on Object-oriented Programming, Systems, Languages, and Applications, Seattle, WA, 2002. ACM Press: New York, NY, 2002; 161-172. DOI: 10.1145/582419.582436.

14. Eckel B. Thinking in Java (4th edn). Prentice-Hall: Englewood Cliffs, NJ, 2006.

15. Miles R. AspectJ Cookbook. O'Reilly: Sebastopol, CA, 2005.

16. Tourwé T, Brichau J, Gybels K. On the existence of the AOSD-evolution paradox. Workshop on Software-engineering Properties of Languages for Aspect Technologies at AOSD 2003, Boston, MA, 2003.

17. Koppen C, Störzer M. PCDiff: Attacking the fragile pointcut problem. Proceedings of the Interactive Workshop on Aspects in Software, Berlin: Germany, 2004.

18. Wloka J. Aspect-aware refactoring tool support. Proceedings of the Workshop on Linking Aspect Technology and Evolution at AOSD 2005, Chicago, IL, 2005.

19. Wloka J. Towards tool-supported update of pointcuts in AO refactoring. Proceedings of the Workshop on Linking Aspect Technology and Evolution Revisited at AOSD 2006, Bonn, Germany, 2006.

20. Monteiro MP, Fernandes JM. Refactoring a Java code base to AspectJ: An illustrative example. Proceedings of the IEEE International Conference on Software Maintenance, Budapest, Hungary, 2005. IEEE Computer Society Press: Los Alamitos, CA, 2005; 17-26. DOI: 10.1109/ICSM.2005.75.

21. Griswold WG. Program restructuring as an aid to software maintenance. PhD Thesis, University of Washington, 1991.

22. Opdyke WF. Refactoring object-oriented frameworks. PhD Thesis, University of Illinois at Urbana-Champaign, IL, 1992.

23. Opdyke WF, Johnson RE. Refactoring: An aid in designing application frameworks and evolving object-oriented systems. Proceedings of the Symposium on Object-oriented Programming Emphasizing Practical Applications, Poughkeepsie, NY, $1990 ; 145-160$.

24. Griswold WG, Notkin D. Automated assistance for program restructuring. ACM Transactions on Software Engineering and Methodology 1993; 2(3):228-269. DOI: 10.1145/152388.152389.

25. Wake W. Refactoring Workbook. Addison-Wesley: Reading, MA, 2005.

26. Elrad $\mathrm{T}$ (moderator) with panelists Aksit M, Kiczales G, Lieberherr K, Ossher H. Discussing aspects of AOP. Communications of the ACM 2001; 44(10):33-38. DOI: 10.1145/383845.383854.

27. Filman RE, Elrad T, Clarke S, Aksit M. (eds.). Aspect-oriented Software Development. Addison-Wesley: Reading, MA, 2005.

28. Laddad R. AspectJ in Action-Practical Aspect-oriented Programming. Manning: Greenwich, CT, 2003.

29. Colyer A. AspectJ. Aspect-oriented Software Development, Filman RE, Elrad T, Clarke S, Aksit M (eds.). Addison-Wesley: Reading, MA, 2005; 123-143.

30. Kiczales G, Hilsdale E, Hugunin J, Kersten M, Palm J, Griswold WG. Getting started with AspectJ. Communications of the ACM 2001; 44(10):59-65. DOI: 10.1145/383845.383858.

31. Martin M, Livshits B, Lam MS. Finding application errors and security flaws using PQL: A program query language. Proceedings of the 20th Annual ACM Conference on Object-oriented Programming, Systems, Languages, and Applications, San Diego, CA, 2005. ACM Press: New York, NY, 2005; 365-383. DOI: 10.1145/1094811.1094840.

32. Filman RE, Friedman DP. Aspect-oriented programming is quantification and obliviousness. Aspect-oriented Software Development, Filman RE, Elrad T, Clarke S, Aksit M (eds.). Addison-Wesley: Reading, MA, 2005; 21-35.

33. Garcia A, Sant'Anna C, Figueiredo E, Kulesza U, Lucena C, Staa A. Modularizing design patterns with aspects: A quantitative study. Transactions on Aspect-oriented Software Development (Lecture Notes in Computer Science, vol. 3880), Rashid A, Aksit M (eds.). Springer: Berlin, Germany, 2006; 36-74. DOI: 10.1007/11687061_2. 
34. Hannemann J, Murphy G, Kiczales G. Role-based refactoring of crosscutting concerns. Proceedings of the 4th International Conference on Aspect-oriented Software Development, Chicago, IL, 2005. ACM Press: New York, NY, 2005; 135-146. DOI: $10.1145 / 1052898.1052910$.

35. Hannemann J. Aspect-oriented refactoring: Classification and challenges. LATEr Workshop at AOSD 2006, Bonn, Germany, March 2006.

36. van Deursen A, Marin M, Moonen L. A systematic aspect-oriented refactoring and testing strategy, and its application to JHotDraw. Technical Report SEN-R0507, Centrum voor Wiskunde en Informatica, 2005.

37. Fuentes L, Jimenez D, Pinto M. Experiences refactoring ambient intelligence applications with aspects. LATE Workshop at AOSD 2005, Chicago, IL, 2005.

38. Kulesza U, Sant'Anna C, Lucena C. Refactoring the JUnit framework using aspect-oriented programming. Companion to the 20th Annual ACM SIGPLAN Conference on Object-oriented Programming, Systems, Languages, and Applications, San Diego, CA, 2005. ACM Press: New York, NY, 2005; 136-137. DOI: 10.1145/1094855.1094901.

39. Nordberg II IM. Aspect-oriented dependency management. Aspect-oriented Software Development, Filman RE, Elrad T, Clarke S, Aksit M (eds.). Addison-Wesley: Reading, MA, 2005; 557-584.

40. Mezini M, Ostermann K. Untangling crosscutting models with Ceaser. Aspect-oriented Software Development, Filman RE, Elrad T, Clarke S, Aksit M (eds.). Addison-Wesley: Reading, MA, 2005; 165-199.

41. Lesiecki N. Enhance Design Patterns with AspectJ, Part 2 (AOP@Work Series at Developerworks), IBM, 2005. http://www-128.ibm.com/developerworks/java/library/j-aopwork6/index.html [25 April 2007].

42. Lagaisse B, Joosen W. Decomposition into elementary pointcuts: A design principle for improved aspect reusability. SPLAT! Workshop at AOSD 2006, Bonn, 2006

43. Kellens A, Gybels K. Issues in performing and automating the 'extract method calls' refactoring. SPLAT! Workshop at AOSD 2005, Chicago, IL, 2005.

44. Tonella P, Ceccato M. Migrating interface implementation to aspects. Proceedings of 20th IEEE International Conference on Software Maintenance, Chicago, IL. IEEE Computer Society Press: Los Alamitos, CA, 2004; 220-229. DOI: 10.1109/ICSM.2004.1357806.

45. Bruntink M, van Deursen A, Tourwé T. Isolating idiomatic crosscutting concerns. Proceedings of the 21st IEEE International Conference on Software Maintenance, Budapest, Hungary, 2005. IEEE Computer Society Press: Los Alamitos, CA, 2005; 322-329. DOI: 10.1109/ICSM.2005.57.

46. Colyer A, Clement A. Large-scale AOSD for middleware. Proceedings of the 3rd International Conference on Aspect-oriented Software Development, Lancaster, U.K., 2004. ACM Press: New York, NY, 2004; 56-65. DOI: $10.1145 / 976270.976279$.

47. Zhang C, Jacobsen H. Re-factoring middleware systems: A case study. Proceedings of the International Symposium on Distributed Objects and Applications, Catania, Italy, 2003 (Lecture Notes in Computer Science, vol. 2888), Meersman R, Tari Z, Schmidt DC (eds.). Springer: Berlin, Germany, 2003; 1243-1262.

48. Cole L, Borba P. Deriving refactorings for AspectJ. Proceedings of the 4th International Conference on Aspect-oriented Software Development, Chicago, IL, 2005. ACM Press: New York, NY, 2005; 123-134. DOI: 10.1145/1052898.1052909. 